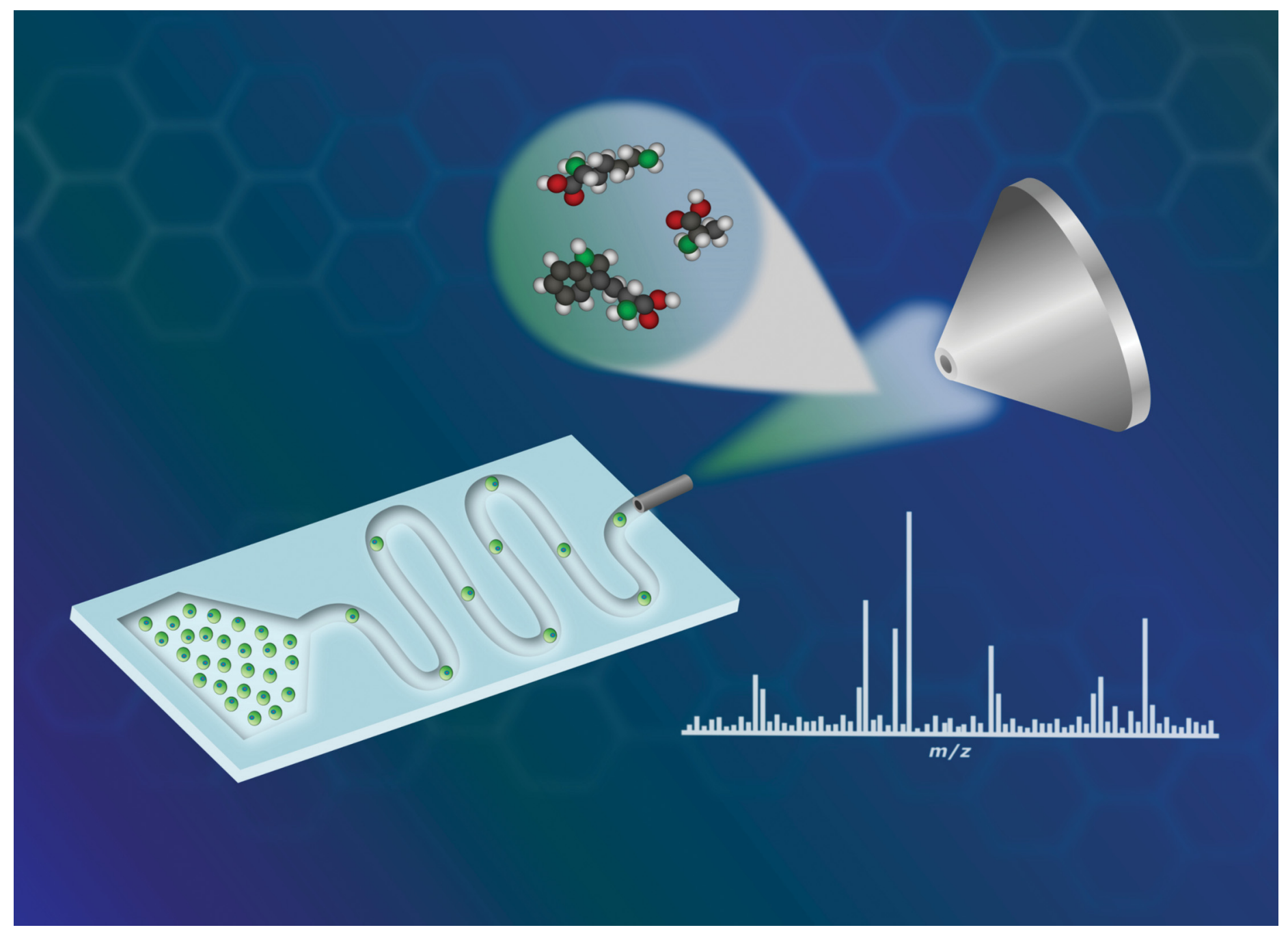

\section{Showcasing research from Dr Trent Northen's laboratory, Lawrence Berkeley National Laboratory, Berkeley, CA, USA}

Faster, better, and cheaper: harnessing microfluidics and mass spectrometry for biotechnology

Northen et al. reviewed recent microfluidic approaches that have been successfully integrated with mass spectrometry analysis for high-throughput bioassays. Microfluidic approaches enable rapid manipulation of small volumes and have found a wide range of applications in biotechnology. The integration of mass spectrometry with microfluidics has the potential to create high-throughput, sensitivity, and specificity assays. This review introduces the major ionization techniques of mass spectrometry that have been integrated with various microfluidic systems. In addition, this work discusses recent high-impact applications and shares future outlooks on multiple aspects of these technologies.

\section{As featured in:}

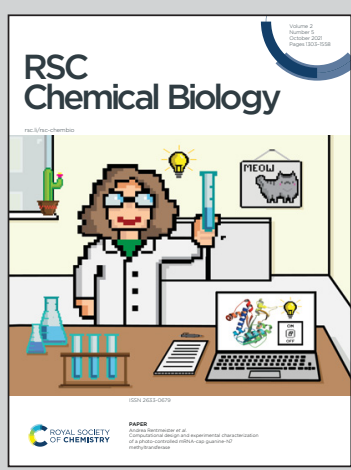

See Trent R. Northen et al., RSC Chem. Biol., 2021, 2, 1331. 


\section{D) Check for updates}

Cite this: RSC Chem. Biol., 2021, 2, 1331

Received 18th May 2021,

Accepted 1st July 2021

DOI: 10.1039/d1cb00112d

rsc.li/rsc-chembio

\title{
Faster, better, and cheaper: harnessing microfluidics and mass spectrometry for biotechnology
}

\author{
Noel S. Ha, (D) ${ }^{\text {ab }}$ Markus de Raad, (D) ${ }^{c}$ La Zhen Han, (D) ${ }^{c d}$ Amber Golini, ${ }^{c d}$ \\ Christopher J. Petzold (iD ${ }^{\text {ab }}$ and Trent R. Northen*abcd
}

\begin{abstract}
High-throughput screening technologies are widely used for elucidating biological activities. These typically require trade-offs in assay specificity and sensitivity to achieve higher throughput. Microfluidic approaches enable rapid manipulation of small volumes and have found a wide range of applications in biotechnology providing improved control of reaction conditions, faster assays, and reduced reagent consumption. The integration of mass spectrometry with microfluidics has the potential to create highthroughput, sensitivity, and specificity assays. This review introduces the widely-used mass spectrometry ionization techniques that have been successfully integrated with microfluidics approaches such as continuous-flow system, microchip electrophoresis, droplet microfluidics, digital microfluidics, centrifugal microfluidics, and paper microfluidics. In addition, we discuss recent applications of microfluidics integrated with mass spectrometry in single-cell analysis, compound screening, and the study of microorganisms. Lastly, we provide future outlooks towards online coupling, improving the sensitivity and integration of multi-omics into a single platform.
\end{abstract}

\section{Introduction}

High-throughput screening (HTS) is a critical step in drug discovery and an important tool for elucidating gene and protein function. ${ }^{1,2}$ It is widely used across pharmaceutical and biotechnological applications with key applications including protein characterization, disease and health monitoring, synthetic biology, and drug development. ${ }^{3}$ High-throughput screening is traditionally defined as the rapid analysis of samples, exceeding $10^{3}$ samples per day. ${ }^{4-6}$ As technology advances, the screening of a large population of biological entities for a particular metabolite, enzyme, protein, nucleic acid, phenotype, or mutation is becoming a significant challenge. Primary difficulties in accomplishing this include the high costs and amount of time required to refine large libraries to a limited number of candidates for further characterization. Therefore, there are three important goals to achieve in HTS: (1) high speed analysis with low-cost operations, (2) high specificity, and (3) high sensitivity for precise measurements.

\footnotetext{
${ }^{a}$ Biological Systems and Engineering, Lawrence Berkeley National Laboratory, Berkeley, CA, USA. E-mail: trnorthen@lbl.gov

${ }^{b}$ US Department of Energy Joint BioEnergy Institute, Emeryville, CA, USA ${ }^{c}$ Environmental Genomics and Systems Biology, Biosciences, Lawrence Berkeley National Laboratory, Berkeley, CA, USA

${ }^{d}$ US Department of Energy Joint Genome Institute, Berkeley, CA, USA
}

Mass spectrometry (MS) is a label-free detection technique and has become a method of choice for high-throughput assays. $^{7,8}$ Currently, mass spectrometry is heavily utilized in many research areas due to its high selectivity based on analysis of a characteristic mass to charge ratio $(\mathrm{m} / \mathrm{z})$ of analytes and their fragments using tandem mass spectrometry (MS/MS). The range of platforms available for mass spectrometry, based on a diverse set of ionization techniques, enables the analysis of a broad range of sample types. ${ }^{9-12}$ However, traditional liquid chromatography-mass spectrometry (LC/MS) approaches for screening samples are time consuming due to chromatographic separation and require relatively large sample volumes, which often makes them cost prohibitive for HTS efforts. While this review paper is focused on integration of MS with microfluidics, it is important to note that there are a range of other label-free analytical methods that can also be considered, including surface-based sensing techniques such as surfaceplasmon resonance (SPR $)^{13,14}$ and surface enhanced Raman spectroscopy (SERS). ${ }^{15,16}$ The reader is referred to several excellent review papers on these topics. ${ }^{17-20}$

Microfluidics is widely used in biotechnology and is central to next-generation sequencing technologies..$^{21-23}$ Within the field of proteomics, there is substantial interest in the integration of microfluidic technologies to process proteins to peptides and carry out the analysis of low samples of low abundance, including those associated with single cells. ${ }^{24} \mathrm{~A}$ range of 
approaches are used to enable manipulation of droplets containing picoliter to nanoliter volumes. ${ }^{25-29}$ Together, such methods provide the capability to rapidly process millions of samples, multiplex numerous processes in parallel, and improve sensitivity even for a low abundance sample by increasing the concentration. These properties of microfluidics, when coupled with mass spectrometry systems, provide potential for high sensitivity and specificity, as well as throughput analysis. ${ }^{30,31}$ Thanks to recent substantial developments in microfluidic technologies, miniaturized microfluidic ion sources have been a very important development for mass spectrometry analyses. ${ }^{32,33}$ The further integration of droplet microfluidic approaches with mass spectrometry has tremendous potential to increase the throughput of the system, which is important for a wide range of important applications. In this review, we introduce the key microfluidic and mass spectrometry approaches and describe the latest applications of coupling microfluidics with mass spectrometry.

\section{Introduction to relevant microfluidic approaches}

Microfluidics is a rapidly developing field and is currently regarded as a critical component to various life sciences. ${ }^{34}$ For instance, it has been well established that microfluidic devices provide numerous advantages for biochemical assays as well as the synthesis of pharmaceuticals..$^{30,31,35}$ In particular, the scale of microfluidic devices enables improved control of reaction conditions, leading to faster, higher-yield production and reduced reagent consumption and system cost. ${ }^{36,37}$ Various types of microfluidic

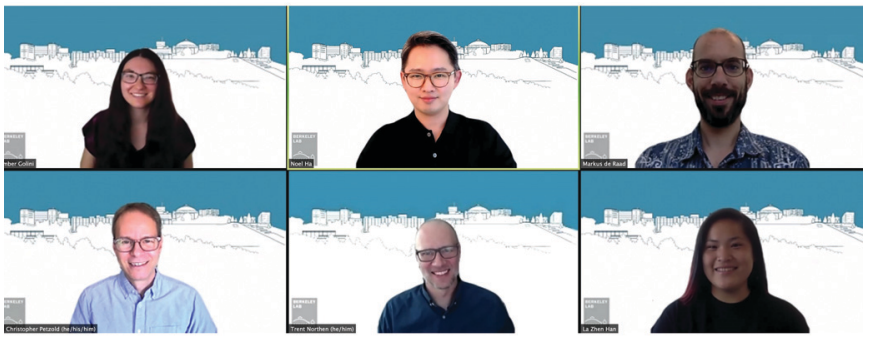

Virtual group photo: Amber Golini (Top left), Noel Ha (Top center), Markus de Raad (Top right), Christopher Petzold (Bottom left), Trent Northen (Bottom center), La Zhen Han (Bottom right)
Dr. Ha obtained his BS in Materials Science at Sung Kyun Kwan University followed by a MS in Biomedical Engineering from the University of California, Irvine and a PhD in Bioengineering from the University of California, Los Angeles with Prof. Michael van Dam. He is currently a post-doctoral fellow at Lawrence Berkeley National Laboratory within the Biological Systems and Engineering Division (BSE) and working with Dr. Trent Northen. Dr. Ha's research is focused on the development of microfluidic bioanalytical technologies for high-throughput quantitative bioassays in a wide range of applications including drug discovery and synthetic biology.

Dr. de Raad earned his masters degree in Drug Innovation from Utrecht University followed by a PhD in Pharmaceutics from Utrecht University with Prof. Enrico Mastrobattista. He then did his postdoctoral research at Lawrence Berkeley National Laboratory in mass spectrometry with Dr. Trent Northen. He is currently a Scientist within the Environmental Genomics and Systems Biology Division (EGSB) in the Northen Lab. His research is focused on developing and applying mass spectrometry based high-throughput screening for unraveling microbial interactions and studying microbial gene function in microbiomes.

La Zhen Han obtained a BA from Sarah Lawrence College and is currently a Research Assistant at the Northen Lab, where she contributes to projects within the Environmental Genomics and Systems Biology Division (EGSB) and Joint Genome Institute (JGI). Her work includes liquid chromatography-mass spectrometry (LCMS) sample processing and instrumentation.

Amber Golini earned her BS in Environmental Management and Protection with a minor in Biology from California Polytechnic State University, San Luis Obispo. She is currently a Research Assistant within the Environmental Genomics and Systems Biology Division (EGSB) and Joint Genome Institute (JGI) in the Northen Lab. She assists with the preparation, processing, and analysis of samples for metabolomics projects using liquid chromatography-mass spectrometry (LCMS) based methods, as well as operating and maintaining LCMS instrumentation systems.

Dr. Petzold obtained his BS in Chemistry and BA in Physics at Taylor University followed by a PhD in Analytical Chemistry from Purdue University with Prof. Hilkka Kenttämaa. He then did post-doctoral research at the University of California, Berkeley in bioanalytical mass spectrometry with Prof. Julie Leary. Dr. Petzold joined Prof. Jay Keasling's lab at the University of California, Berkeley in 2005 and joined Berkeley Lab in 2008. He is currently a Staff Scientist within the Biological Systems and Engineering Division (BSE). Dr. Petzold's research is focused on the development of high-throughput analytical methods and quantitative proteomics.

Dr. Northen obtained his BS in Chemical Engineering at the University of California Santa Barbara followed by a PhD in Chemistry and Biochemistry from Arizona State University with Prof. Neal Woodbury. He then did a Post-Doctoral Fellow at the Scripps Research Institute in mass spectrometry and metabolomics with Prof. Gary Siuzdak. Dr. Northen started his lab at Berkeley Lab in 2008 and is currently Deputy Division Director and a Senior Scientist within the Environmental Genomics and Systems Biology Division (EGSB). The Northen group's research is focused on using exometabolomics to link genomes with environments to understand how webs of microbes cycle carbon and sustain biomes. 
devices have been coupled to mass spectrometry platforms to manipulate samples (including processes such as cell lysis, separation, purification, and step-wise chemical and biological reactions), ${ }^{32,38-40}$ and here we will discuss the most frequently reported types of coupling that have emerged over the past four years.

\section{Continuous-flow system}

The most common type of microfluidic device is a continuous-flow system where the flow through the microchannel is driven by pressure. A variety of pressure sources can be used to control the flow in this type of system. The Quake group pioneered this type of system and developed high-density microfluidic chips that contain plumbing networks with thousands of micromechanical valves and hundreds of individual chambers ${ }^{41,42}$ (Fig. 1A). This setup is capable of automating hundreds of thousands of experiments in parallel in a single device. Continuous-flow microfluidic devices have been extensively coupled with mass spectrometry as it is particularly useful to automate the multiple steps of sample pretreatment (e.g., cell lysis, protein extraction, purification, desalting, etc.) prior to mass spectrometry analysis. ${ }^{43-45}$

\section{Microchip electrophoresis}

Another type of continuous-flow system is driven by an electric field (rather than pressure) via capillary electrophoresis (CE) (Fig. 1B). ${ }^{46} \mathrm{CE}$ is a separation technique with high separation efficiency and low consumption of sample and reagents. ${ }^{4748}$ $\mathrm{CE}$ is employed in diverse applications including DNA and protein separation, ${ }^{49-51}$ detection of disease biomarkers, ${ }^{52,53}$ environment monitoring, ${ }^{54,55}$ and pharmaceutical analysis. ${ }^{48,56-59}$ Furthermore, it can readily be miniaturized using microfluidic chip technology, called microchip electrophoresis (MCE). MCE has been used for high-resolution separations where portability or small sample volumes are especially important and it has been commonly coupled with electrospray ionization mass spectrometry (ESI-MS). ${ }^{35,60,61}$ The MCE system requires the use of high voltage for operation, which makes parallel sample processing challenging without the use of multiple devices simultaneously.

\section{Droplet microfluidics}

Droplet microfluidic (DMF) systems compartmentalize reactants through the use of an inert carrier fluid (typically oil) to encapsulate aqueous samples in droplets. (Fig. 1C). ${ }^{62}$ These systems can produce droplets in the volume range of $0.05 \mathrm{pL}$ to $1 \mathrm{~nL}$ (i.e., $5-120 \mu \mathrm{m}$ in diam.) instead of the microliter volumes commonly used in conventional methods. ${ }^{26}$ Droplets can encapsulate cells, DNA, or other molecules inside the aqueous phase without risking cross-contamination, and manipulation and measurement of droplets at $\mathrm{kHz}$ speeds enable up to $10^{8}$ samples to be screened in one day. ${ }^{26,63}$ This method of droplet microfluidics can increase assay sensitivity by raising the effective concentration of low abundance species and decreasing the time required to reach reaction equilibrium and detection thresholds. When coupled with next-generation sequencing or MS, droplet microfluidics enables high-throughput screening applications such as single-cell and single-molecule assays that are currently unfeasible or impossible with conventional methods. ${ }^{64,65}$ However, controlled droplet manipulation for downstream mass spectrometry analysis can be challenging, and droplet stability over a longer period at an elevated temperature can become an issue. When coupled with mass spectrometry, the carrier oil phase used in the droplet

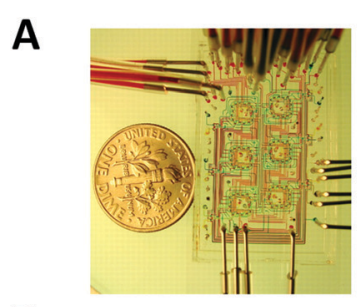

D

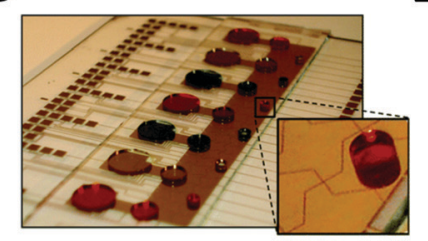

B

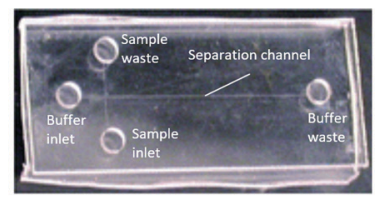

E

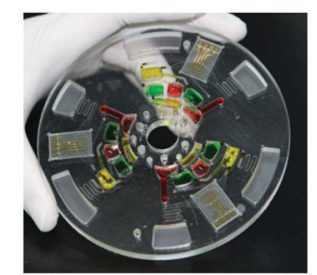

C

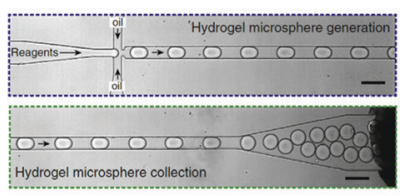

$\mathbf{F}$

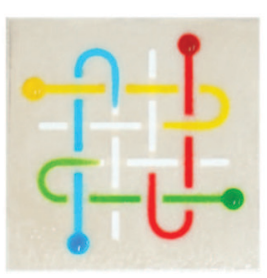

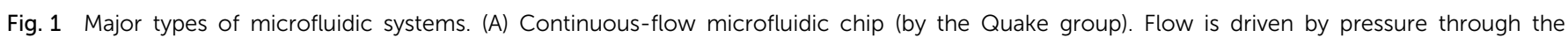

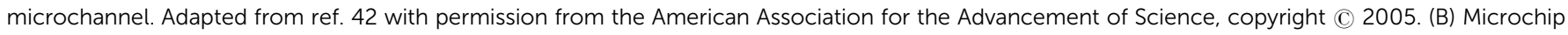

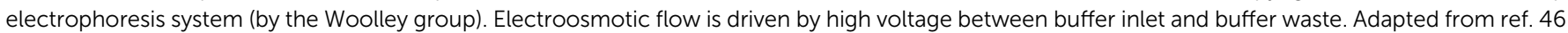

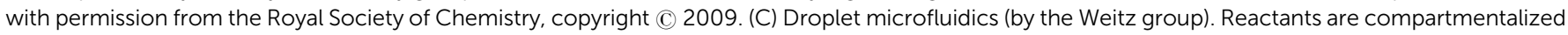

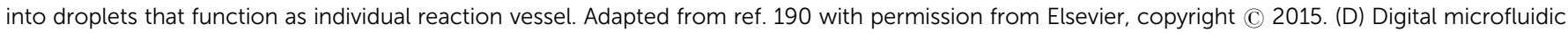

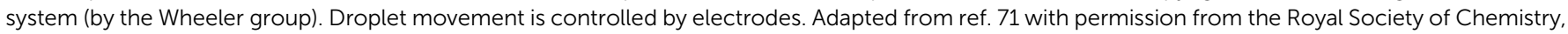

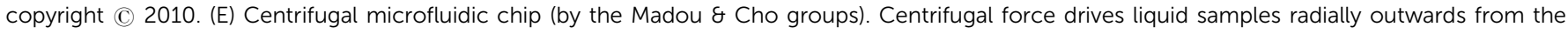

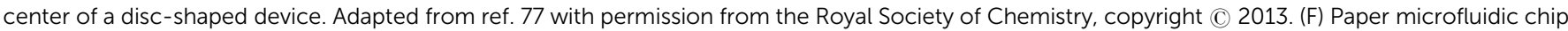

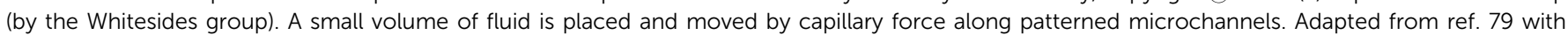
permission from the National Academy of Sciences, copyright (C) 2008. 
microfluidics can degrade the stability, efficiency, and accuracy of mass spectrometry detection and the sample transportation rates might not sync with the MS data acquisition rate for transient signal acquisition. ${ }^{66}$ Optionally, the droplet contents can be extracted from the oil phase to an aqueous channel for subsequent ionization. ${ }^{67-69}$

\section{Digital microfluidics}

Digital microfluidics is a developing liquid-handling technology that utilizes electrodes to individually control droplets. ${ }^{70}$ These microliter-sized droplets (typically larger than ones used in aforementioned droplet microfluidics) can be made to move, merge, and split across the array (Fig. 1D). ${ }^{71}$ Because of its unique advantages (such as programmable control of sample handling and flexible device geometry) digital microfluidics has been applied to a wide range of fields. ${ }^{72-74}$ Digital microfluidics is a particularly useful option for step-wise sample pretreatments in chemical and biological reactions (such as cell lysis and protein extraction) prior to mass spectrometry analysis. ${ }^{75}$ However, device fabrication is costly and complicated. In addition, the device is prone to failure at high reaction temperature or high voltage.

\section{Centrifugal microfluidics}

In centrifugal microfluidics, centrifugal forces induced on the sample drive the liquids radially outwards from the center of a disc-shaped device (Fig. 1E), ${ }^{76,77}$ and the flow of fluids in a centrifugal platform has been well characterized. ${ }^{78}$ In comparison to other systems, the instrumentation demands of centrifugal microfluidics are much lower. The only required component is a simple, compact motor to create the forces needed for fluid manipulation, thereby eliminating any complex tubing or external pressure systems. Centrifugal microfluidic chips can also be mass-produced using inexpensive materials, making them lowcost and suitable for disposable devices. However, large-scale integration is challenging, as throughput is often limited to a single centrifuge. Furthermore, contact-free on-line interface with downstream mass spectrometry analysis is not currently available.

\section{Paper microfluidics}

Microfluidic paper-based analytical devices ( $\mu$ PADs) or lateralflow microfluidic systems are made of patterned paper on which a small volume of fluid is placed and moved by capillary force for subsequent chemical or biological reactions (Fig. 1F) ${ }^{79}$ The typical readout can be obtained from a colorimetric assay, but other sophisticated detection modalities such as electrochemical and mass spectral detection have been also explored. Paper is not only inexpensive, but it is also a very light substrate that can be stored and transported easily. The detailed outstanding features of paper-based microfluidic devices were summarized elsewhere. ${ }^{80}$ Many paper microfluidic devices have been developed in an attempt to make diagnostic devices environmentally friendly and affordable. $\mu$ PADs are also the subject of development for rapid testing (for example, diagnosis of the SARS-CoV-2 virus) in urgent situations or remote areas where more complex and expensive technologies are unavailable. ${ }^{81}$ However, $\mu$ PADs have limitations related to the material properties of paper, the fabrication techniques used, and the detection methods connected to the devices. ${ }^{80}$ Specifically, the sample retention within paper-fluidic channels and the sample evaporation during transport can result in a low efficiency of sample delivery within the device, which in turn can negatively impact downstream mass spectrometry analysis. Furthermore, some hydrophobic agents used to pattern devices fail to build barriers strong enough to repel low surface tension samples.

\section{Coupling of microfluidics with mass spectrometry}

Microfluidic chips have been coupled with mass spectrometry via various interfaces and ionization methods. Electrospray ionization (ESI) and matrix-assisted laser desorption ionization (MALDI) are currently the most popular techniques coupled with microfluidics. More recently, other ionization techniques have also been coupled with mass spectrometry, including surface-assisted laser desorption ionization mass spectrometry (SALDI), desorption electrospray ionization (DESI), inductively coupled plasma (ICP), and surface acoustic wave nebulization (SAWN).

\section{A. Electrospray ionization}

Electrospray ionization (ESI) is the most common ionization method coupled with microfluidic systems. In ESI, a fluid is pumped through a capillary or channel and subjected to an electric field, producing a Taylor cone, which ejects charged droplets that are desolvated and introduced into the mass spectrometry system. The range of microfluidic devices integrated with ESI-MS is discussed below.

Continuous flow microfluidics \& ESI-MS. Continuous-flow microfluidic devices are suited for experiments where sample pretreatment prior to mass spectrometry analysis is required. ${ }^{44}$ In conventional liquid chromatography-mass spectrometry (LC/MS) analysis, chromatographic separation is performed prior to mass spectrometry analysis. Sample cleanup methods like solid-phase extraction (SPE) are often utilized to remove interfering species prior to sample ionization. On-chip liquid chromatography has been explored extensively for separation of analytes from complex biological matrices prior to MS analysis. $^{82,83}$ Recently, a new type of commercial microchip chromatography cartridge equipped with a micropillar array-based column ( $\mu \mathrm{PAC}^{\mathrm{TM}}$, PharmaFluidics) has been introduced. ${ }^{84,85}$ However, the microchip liquid chromatography typically requires an expensive and bulky high-pressure source to overcome high fluidic resistance through a microfluidic column. SPE can be implemented during off-line sample preparation prior to mass spectrometry analysis ${ }^{86}$ or seamlessly through an on-line format. ${ }^{43,45}$ In SPE, samples are loaded onto a packed bed of sorbent and the bed is rinsed to remove poorly retained species, after which a solvent is passed through the bed to elute analytes. Several groups have developed microfluidic SPE-based 
purification systems for coupling with ESI-MS. For instance, Lin et al. developed an integrated microfluidic device with three individual components (cell co-culture, protein detection, and pretreatment for drug metabolites) to probe interactions between tumor and endothelial cells (Fig. 2A). ${ }^{44}$ The SPE component of the system was loaded with C-18 particles (45 $\mu \mathrm{m}$ in diam.) on a chip and metabolites from drug-treated cocultured cervical carcinoma cells (CaSki cells) and human umbilical vein endothelial cells (HUVECs) were analyzed via MS. Gasilova et al. reported microfluidic on-line sample preconcentration and purification using C8-functionalized mesoporous magnetic microspheres as a SPE sorbent prior to MS analysis. ${ }^{43}$ A magnetic field was applied to ensure the selective enrichment of large hydrophobic peptides $(2.5-7 \mathrm{kDa})$ and within less than 35 minutes the system provided $66.5 \%$ of protein sequence coverage from $75 \mathrm{fmol}$ of BSA tryptic digest.

Microchip electrophoresis \& ESI-MS. Microfluidic capillary electrophoresis is widely utilized for separation and purification of samples prior to mass spectrometry analysis. MCE is most frequently coupled with ESI due to its compatible flow rate and the high voltage used for both separation and ionization. ${ }^{62,87-91}$ MCE systems with integrated nano-electrospray ionization (nano-ESI) interfaces are also commercially available for easy coupling to MS. Scholl et al. reported a new approach for the sheathless coupling of MCE with ESI-MS (Fig. 2B) to replace conventional approaches where sheath-flow is required to focus the stream of ionized sample at the end of the electrophoresis channel. ${ }^{87}$ This is done through the use of an ion-conductive hydrogel membrane, which is placed between a primary electrophoretic separation channel and a supporting channel. A reliable electrical connection is then established between the coupled systems without sacrificing separation performance. Moreover, this sheathless coupling reduced sample dilution and loss that commonly occur due to the sheath fluid. The measurement of each sample took $\sim 10-30 \mathrm{~s}$.

Droplet microfluidics \& ESI-MS. Droplet microfluidics interfaced with ESI-MS provides a label-free HTS platform. The first high-throughput droplet-mass spectrometry method was introduced by the Kennedy group, who demonstrated a screening with throughput as high as 1.7 samples per s. ${ }^{92}$ Following this, Steyer et al. developed a platform for the analysis of droplets by nano-ESI, which is an attractive approach for droplet analysis since it allows rapid analysis with high mass sensitivity and resistance to matrix effects. ${ }^{93}$ Continuous infusion to a nanoESI emitter from a microfluidic chip was conducted for as long as 2.5 hours, facilitating the analysis of over 20000 samples (Fig. 2C). The signal was stable for droplets as small as $65 \mathrm{pL}$ and for throughputs up to 10 droplets per s. Wink et al. developed a more advanced droplet microfluidic system for the analysis of secondary metabolites produced inside the droplets through simultaneous fluorescence imaging and ESI-MS analysis. For this method, microbes were encapsulated in $\sim 200 \mathrm{pL}$ droplets; long-term incubation was performed on one chip before the droplets were transferred to a second microfluidic chip for MS analysis. Fluorescent markers were monitored before the droplets were ionized, which enabled analysis of metabolites and fluorescent labels in a complex biological matrix. They also demonstrated the detection of streptomycin produced in situ by ESI-MS using Streptomyces griseus hyphae. Recently, the Belder group has even explored the integration of droplet microfluidics with on-chip liquid chromatography for the separation of analytes for the

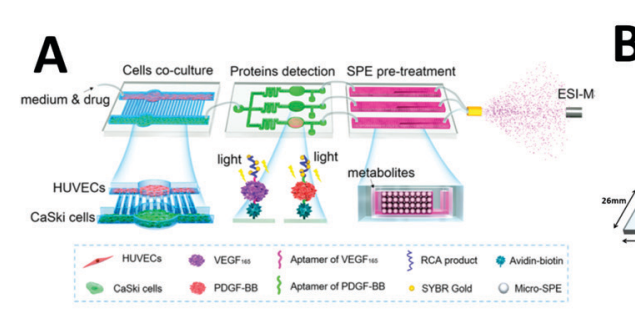

B C

D

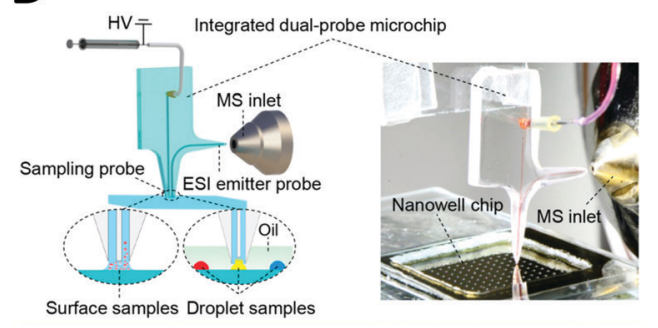

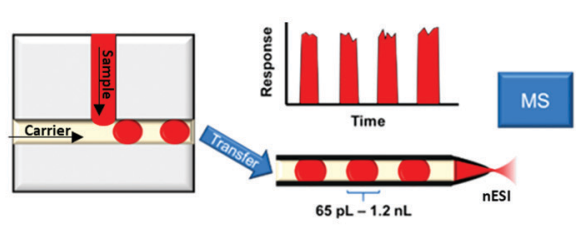

E

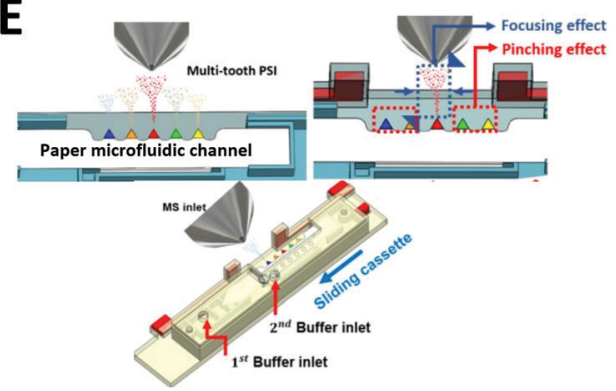

Fig. 2 Coupling of microfluidics with electrospray ionization mass spectrometry (ESI-MS). (A) Continuous flow microfluidic chip with multiple functions coupled with ESI-MS. Adapted from ref. 44 with permission from the American Chemical Society, copyright (C) 2017. (B) Microchip electrophoresis chip coupled with ESI-MS. Adapted from ref. 87 with permission from Springer Nature, copyright (C) 2018. (C) Droplet microfluidics coupled with nano-ESIMS. Adapted from ref. 93 with permission from the American Chemical Society, copyright (C) 2019 (D) direct surface and droplet micro-sampling probe for downstream ESI-MS. Adapted from ref. 95 with permission from the American Chemical Society, copyright (C) 2017. (E) Paper microfluidic device equipped with paper spray ionization. Adapted from ref. 98 with permission from the IEEE, copyright (C) 2020. 
downstream analysis by ESI-MS. ${ }^{83,94}$ The sample flows through the microfluidic channel packed with chromatographic beads, followed by the droplet generation.

Another method, developed by Huang et al., involves using a microfluidic device for direct surface or droplet micro-sampling followed by ESI. ${ }^{95}$ A single glass microfluidic chip integrates a sampling probe, an electrospray emitter probe, and an on-line mixer (Fig. 2D). Furthermore, two types of sampling probes were developed: a parallel-channel probe for dry spot droplets and a U-shaped channel probe for liquid-phase droplets. The system was demonstrated to be capable of MS analysis of nanoliter-scale chemical reactions. The assay throughput was $\sim 13$ s per droplet, hundreds of times faster than those of conventional LC-MS systems.

When coupling droplet microfluidics with ESI-MS, carryover between droplets that are ionized consecutively needs to be addressed. Other droplet microfluidic systems coupled with ESI-MS have been previously reported. ${ }^{40}$ However, most of them require off-line sample injection for ionization.

Paper spray ionization. One variation of ESI, called paper spray ionization (PSI), was recently introduced for the rapid analysis of biological species in a sample solution. ${ }^{96,97}$ In PSI, an electric field is applied to cut filter paper with absorbed sample solution for direct ESI of the liquid sample and detection by mass spectrometry. The advantages of PSI are similar to those of paper microfluidics: ease-of-use, affordability, and portability. Li et al. developed a paper-based microfluidic device for mass spectrometry analysis of caffeine and nicotine metabolism in urine and hair samples. ${ }^{98}$ The paper-based device contains a main flow channel for sample chromatography to separate the species in the liquid sample and multiple tips for PSI for mass spectrometry analysis (Fig. 2E). It detected the caffeine species in the urine sample and the nicotine/cotinine species in the extracted solution of $2 \mu \mathrm{L}$ from heavy smoker's hairs. These results support that the paper microfluidics combined with the PSI has potential for rapid drug abuse screening test.

When analyzing samples in complex biological matrices by ESI-MS, among other mass spectrometry techniques, ion suppression is a critical issue especially in the analysis of miniscule amounts of metabolites originating from single cells. ${ }^{99}$ Ion suppression is a process where the detection of a given analyte is reduced by the presence of another-for example, salty samples or those containing polyethylene glycol (PEG) surfactants often suffer from this effect. Another challenge for ESI-coupled technologies is the loss of sensitivity over consecutive measurements due to accumulation of materials on the source. When dealing with droplets containing the sample in the respective matrix, the proper choice of surfactants is also crucial as it might reduce the ionization during the electrospray process. ${ }^{100}$

\section{B. Desorption electrospray ionization}

Desorption electrospray ionization (DESI) is an ambient ionization technique which combines features of electrospray and desorption ionization methods. ${ }^{101-103}$ In DESI, charged solvent droplets are directed onto the surface of the sample to be analyzed. The impact of the charged droplets produces ions from the surface that are directed towards the inlet of a mass spectrometer. DESI is the most used technique for ambient mass spectrometry-based bioanalysis since it allows analysis of both solid and liquid samples with little or no sample preparation. This spray-based technique benefits from increased throughput and the ability for spatially resolved molecular features to be probed under ambient conditions. ${ }^{103}$ de Freitas et al. reported the use of DESI mass spectrometry imaging for dried sample on the microfluidic paper-based analytical devices ( $\mu$ PADs) ${ }^{104}$ In paper microfluidics with colorimetric readouts, the formation of color gradients or lack of color uniformity on the detection zone can compromise the readout reliability. L-DESI measurements revealed a heterogeneous distribution of chromogenic agent (iodide and triiodide ions) at the zone edge (Fig. 3A). However, compared to vacuum desorption mass spectrometry, a disadvantage of DESI is lower spatial resolution (approximately 1800-200 $\mu \mathrm{m}$, compared to $30-50 \mu \mathrm{m}$ for MALDI). ${ }^{105}$ Recently, a nano-DESI probe was developed to improve the spatial resolution to approximately $12 \mu \mathrm{m}$. With the nano-DESI probe fabricated using two capillaries, controlled desorption of analytes present in a specific region of specimen has been demonstrated using a small amount of solvent $\left(\sim 0.5 \mu \mathrm{L}\right.$ for each spectrum acquisition). ${ }^{106}$

Liquid desorption electrospray ionization (L-DESI). LiquidDESI, or L-DESI, is the application of DESI on liquid samples.
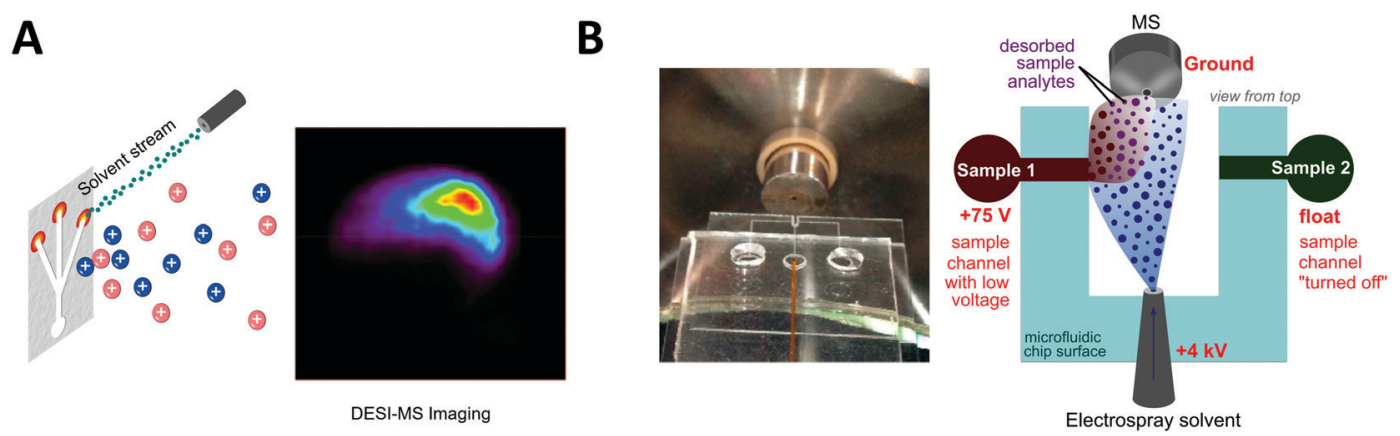

Fig. 3 Coupling of microfluidics with desorption electrospray ionization mass spectrometry (DESI-MS). (A) Microfluidic paper-based analytical device with DESI-MS. Adapted from ref. 104 with permission from the American Chemical Society, copyright (C) 2018. (B) Image of microfluidic voltage-assisted liquid-DESI (left) where the liquid sample is desorbed by the stream of solvent, and a schematic illustration of the working principle (right). Adapted from ref. 107 with permission from the American Chemical Society, copyright (C) 2017. 
The Liu group developed a microfluidic voltage-assisted L-DESI source in a microfluidic format: ${ }^{107}$ their chip has an L-DESI cavity where the charged electrospray solvent droplets are generated and selectively directed towards either side of two independent sample reservoirs (Fig. 3B). A low voltage (+75 V) was applied to either of the two sample reservoirs to form an electrical circuit between the ESI emitter and the exit of desired sample solution. Direct analysis of urine, serum, and cell lysate samples detected compounds of biomedical interest, including nucleosides, monoamines, amino acids, and peptides, with the assay throughput of around 1 min per sample. In following works of the Liu group, the chip geometry has been modified to further improve the ionization efficiency. ${ }^{108,109}$ Voltage-assisted L-DESI-MS/MS techniques have significant potential for direct analysis of biofluids and could potentially be adapted for point-of-care devices.

\section{C. Surface-based laser desorption ionization mass spectrometry}

Laser desorption ionization mass spectrometry (LDI-MS) has been widely used in organic and biological sample analysis. ${ }^{110}$ Of the many LDI-MS methods, matrix-assisted laser desorption ionization mass spectrometry (MALDI-MS) is the most frequently used and has been used to analyze biological samples for protein and bacteria identification, as well as biomarker and metabolite detection. ${ }^{111-113}$ Since LDI is very rapid (nanosecond timescale) these techniques can be extremely highthroughput.

Matrix-assisted laser desorption lonization (MALDI). In MALDI-MS, analytes are co-crystallized with a matrix that absorbs and transfers the laser's energy to the analyte. ${ }^{113}$ Sample and matrix are spotted on the conductive surface, either via manual pipetting or a robotic liquid handler. MALDI-MS has primarily been used to analyze peptides, proteins, and nucleic acids (rather than small molecules) since abundant matrix ions $(<1000 \mathrm{Da})$ can obscure or interfere with small molecule analysis.

MALDI has frequently been coupled with microfluidics, as it allows for automated and high-throughput sample preparation. Thus, microfluidic devices with MALDI-MS can be useful tools to investigate samples in real-time without compromising sample integrity. Filla et al. described a continuous-flow microfluidic device capable of automated cell lysis, metabolite extraction, and quenching of enzymatic activity. ${ }^{114}$ Quenching efficiency was measured with an off-line MALDI-MS assay of exogenous isotopic adenosine triphosphate (ATP) hydrolysis to isotopic adenosine diphosphate (ADP), which was used as a marker of metabolite degradation. The samples processed on the chip were manually transferred onto a MALDI plate for mass spectrometry analysis.

Grant et al. developed a more advanced continuous-flow microfluidic system to directly prepare samples over a MALDI-MS surface. An elastic polydimethylsiloxane (PDMS) microfluidic chip was reversibly clamped against a chemically functionalized gold layer to create a MALDI surface (Fig. 4A). The sample flows through the microfluidic channel while conducting an enzyme reaction. Then, the reaction product is immobilized onto the floor of the microfluidic channel (gold layer) to form a self-assembled monolayer which is later scanned to visualize reaction progress via mass spectrometry analysis.

The Dittrich group previously developed a droplet spotting and analysis platform for MALDI-MS analysis of secreted proteins from single cells. ${ }^{115}$ Droplets containing single cells are spotted onto a custom-made indium tin oxide (ITO)-glass plate and the hydrophilic/hydrophobic pattern on the plate guides droplets to a predefined hydrophilic position. Recently, they developed a new way to split arrayed droplet to extract cell supernatant. ${ }^{116}$ A second plate was placed above the droplet array plate and brought in contact with the droplets (Fig. 4B). All 200 droplets were sampled in parallel by this plate-based droplet splitting and analyzed via MALDI-MS upon drying to detect the protein brazzein secreted from $\sim 500$ cells in each droplet.

Unfortunately, most reported microfluidic systems require manual sample transfer to MALDI plate prior to mass spectrometry analysis. Even in cases where sample arrays are prepared using microfluidic devices, the mass spectrometry surface needs to be manually separated from the microfluidic device before transfer to a mass spectrometry system Thus, a drawback of using MALDI-MS for microfluidic coupling is the difficulty of complete on-line analysis without human intervention. Significant advancements in the microfluidic interface are required for automated on-line analysis.

Surface-assisted laser desorption ionization (SALDI). Even with its popularity, MALDI-MS has several drawbacks, including matrix interference for small molecule analysis and generation of hot spots during the crystallization process of organic matrix. $^{7,110}$ Another LDI-MS technique called surface-assisted laser desorption ionization (SALDI) uses a specific surface or substrate to aid desorption/ionization. Unlike MALDI, SALDI does not require an organic matrix, which can produce high chemical background in the low mass region. ${ }^{110,117}$ SALDI-MS has the additional advantages of easy sample preparation and elimination of hotspots. Due to these advantages, there has been increasing interest in using SALDI-MS for analysis of biological, environmental, and forensic samples. ${ }^{118-121}$ A variety of nanostructure-based SALDI surfaces have been developed ${ }^{122-124}$ including etched silicon wafers with nanostructures e.g., nanostructure-initiator mass spectrometry (NIMS), ${ }^{125}$ black silicon NIMS, ${ }^{126}$ silicon nanopost arrays (NAPA), ${ }^{127-130}$ Nanowires, ${ }^{38}$ and gold nanoparticle (Au NP)-modified surfaces. ${ }^{122,124}$ The reader is referred to a recent review on SALDI-MS for more information. ${ }^{131}$ Despite these advantages, it is important to note that the most reported SALDI-MS methods have not been coupled with microfluidics, and the fabrication of custom surfaces for sample ionization is required, since SALDI surfaces or plates are not commercially available.

However, there have been only a few reports where microfluidic devices were coupled with SALDI-MS, including where a DMF device was used for automated sample handling and followed by SALDI-MS at very low throughput. ${ }^{132}$ Recently, Heinemann et al. developed a higher throughput system that 
A

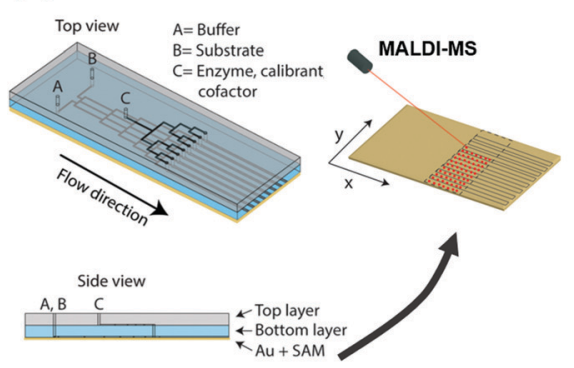

B

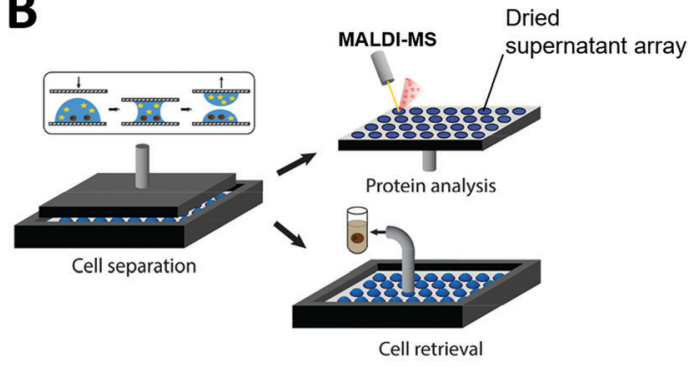

C

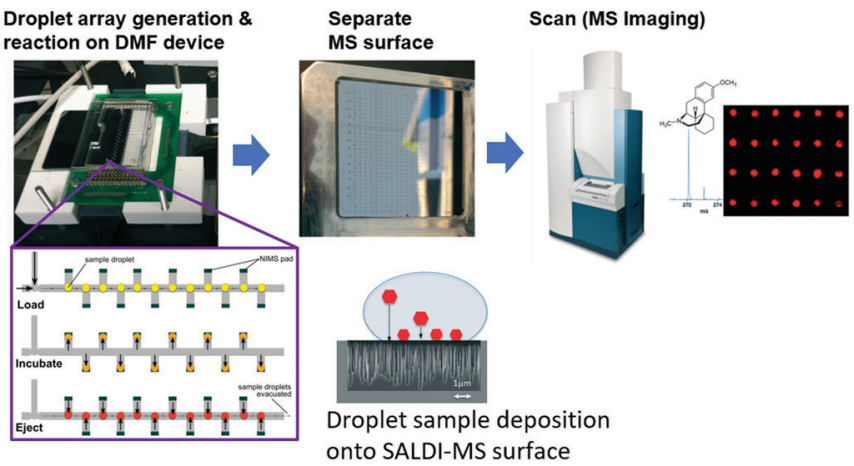

Fig. 4 Coupling of microfluidics with laser desorption ionization mass spectrometry (LDI-MS). (A) Continuous-flow microfluidic chip with a functionalized bottom layer as Matrix Assisted Laser Desorption lonization (MALDI) surface for enzyme reaction (left) and the MALDI-MS scanning of the separated MS surface. Adapted from ref. 191 with permission from the American Chemical Society, copyright (C) 2018. (B) Nanoliter droplet arrays for protein analysis in single yeast cells by MALDI-MS; samples of cell supernatant are taken by parallel plate-based droplet splitting method. Adapted from ref. 116 with permission from the American Chemical Society, copyright (C) 2020. (C) Digital microfluidic (DMF) device interfaced with SALDI-MS surface (nanostructure initiator mass spectrometry, NIMS). Enzyme reactions are performed inside the arrayed droplets, and the mass spectrometry surface with the dried sample array can be separated from the DMF device and scanned for mass spectrometry imaging. Adapted from ref. 133 with permission from the Royal Society of Chemistry, copyright (C) 2017

integrates DMF with NIMS. ${ }^{133}$ Briefly, NIMS uses liquid initiator-coated silicon nanostructures to generate gas phase ions from surface-adsorbed molecules upon laser irradiation. The microfluidic device constructed by Heinemann et al. contains electrodes for droplet manipulation and is patterned with the NIMS array for sample deposition for downstream MS analysis (Fig. 4C). In the study, enzyme reactions were carried out inside droplets using a premixed enzyme reaction solution, and arrayed in discrete locations that had local MS surfaces where sample could be deposited. Once the reaction was completed over the NIMS array, the droplets were removed and the NIMS array was scanned in a commercial MALDI-MS system. The system proved capable of directly measuring the substrates and products of enzymatic reactions and is broadly applicable to many molecular classes including metabolites, drugs, and peptides. This proof-of-principle study reveals SALDI surfaces can be fabricated into an array format for high-throughput microfluidic sample processing. This system can be potentially adapted for diverse array-based compound screening in the future. However, to overcome low sensitivity observed for certain sample types, other sample loading techniques such as complete drying of sample droplets on the MS surface can be considered in the future. Also, complete on-chip sample manipulation, rather than manipulation via premixed solutions, would be desired to automatically screen large compound libraries and possible reaction combinations.

Recently, a centrifugal microfluidic device was coupled with SALDI-MS. Zhao et al. developed a centrifugal microfluidic disc that performs sample cleanup on human serum samples $(\sim 5 \mu \mathrm{L})$ for subsequent metabolite analysis by SALDI-MS. ${ }^{38}$ The disc device consists of six layers of polyester film with a $10 \mathrm{~cm}$ diameter and $100 \mu \mathrm{m}$ thickness per layer. This system demonstrated sufficient removal of proteins, lipids, and other biomolecules for effective downstream MS analysis of multiple small-ion metabolites in the human serum samples. After cleanup in the rotating centrifugal disc, the sample was manually transferred to the SALDI-chip (silicon nanoposts deposited on a silicon wafer) for SALDI-MS analysis. This two-chip system could potentially be integrated into a single device by incorporating the SALDI surface within the chamber in the centrifugal disc for on-line MS analysis.

\section{D. Inductively coupled plasma}

Inductively coupled plasma mass spectrometry (ICP-MS) is a powerful analytical instrument for trace elements detection. ${ }^{134}$ In particular, ICP-MS enables accurate identification and quantification of trace elements and their species in cells. When on-line coupled with IPS-MS, widely used water-in-oil 
droplet microfluidics for single cell analysis encounters several problems. Frequently used carrier oil is not suitable for ICP-MS measurement due to relatively high carbon content. Wang et al. developed a droplet microfluidic chip on-line coupled with ICP-MS that uses a high-viscosity alcohol as a carrier phase to generate single-cell droplets (Fig. 5A). ${ }^{135}$ Droplets were generated at a frequency of $3-6 \times 10^{6}$ droplets $\mathrm{min}^{-1}$, and 2500 single-cell droplets were injected and analyzed per minute. Recently, they used this system to investigate cellular uptake of AuNPs by single HeLa cells as a tool to study intracellular drug delivery and cell/tissue imaging. ${ }^{136}$

Considering cells are already specialized "droplets" with a hydrophilic surface and an elastic hydrophobic membrane, Zhou et al. developed an oil-free passive microfluidic system (OFPMS) for the direct infusion of single cells $(\sim 10 \mu \mathrm{m}$ in diam., pL-range) into a micronebulizer for ICP-MS ${ }^{66}$ (Fig. 5B). This system enables single cell isolation through the use of a thermo-decomposable buffer that eliminates the use of any oil and incompatible polymer carriers. The quantitative single-cell transportation of endogenous zinc ( $\mathrm{Zn}$ ) and exogenous AuNPs in HeLa cells and RAW264.7 macrophages were measured with adjustable throughput ranging from 400 to 25000 cells per min.

\section{E. Acoustic wave nebulization/ionization}

Acoustic-based microfluidics has been developed for use with micro and nanoscale samples and appears to be an effective
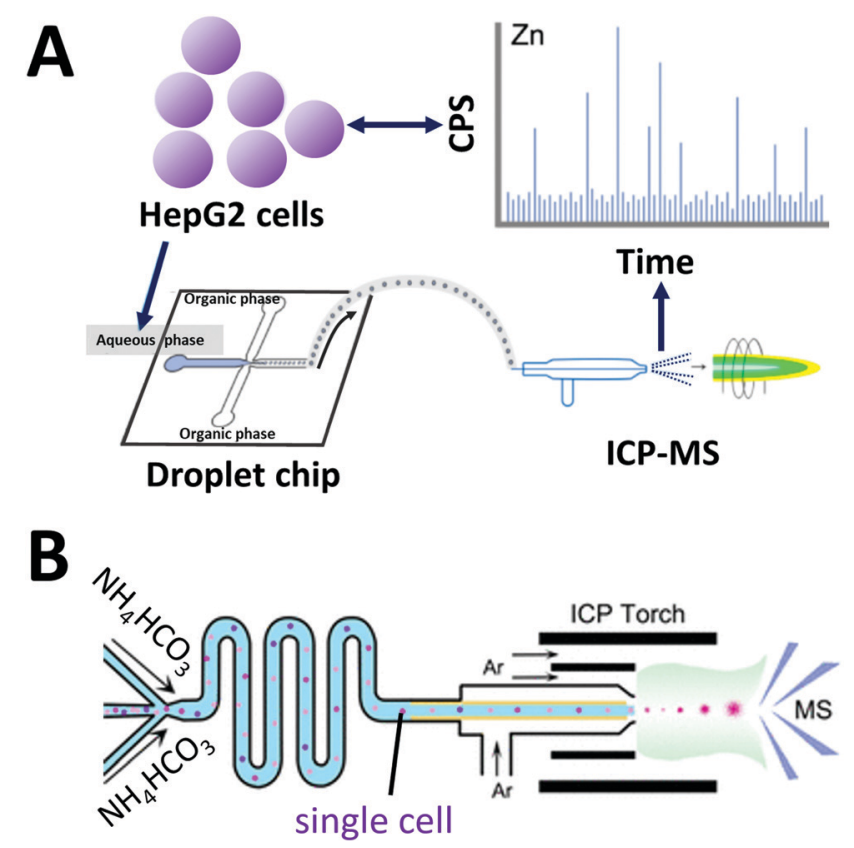

Fig. 5 Coupling of microfluidics with inductively coupled plasma mass spectrometry (ICP). (A) Droplet chip-ICP-MS single-cell analysis system using high viscosity alcohol (alternative to oil phase) to generate single-cell droplets Adapted from ref. 135 with permission from the American Chemical Society, copyright (C) 2017. (B) Direct infusion of single cells with thermo-decomposable buffer as carrier phase into a mass spectrometer via ICP torch. Adapted from ref. 66 with permission from the American Chemical Society, copyright (C) 2020. method of manipulating small volumes. ${ }^{24,137,138}$ With an acoustic-based approach, researchers utilize gentle pressure waves to control a minute amount of sample in a contactless and precise manner for numerous research and industrial applications. ${ }^{139}$ A technique called surface acoustic wave nebulization (SAWN) utilizes standing waves to ionize droplet samples on a surface in an ambient environment at atmospheric pressure. ${ }^{24,137,140,141}$ SAWN was pioneered by Goodlett et al. as a microfluidic interface for mass spectrometry. ${ }^{24,142,143} \mathrm{In}$ SAWN, the surface acoustic wave is generated through electrodes embedded on a dielectric chip, onto which a liquid sample droplet is placed. The acoustic energy transferred to the droplet overcomes its surface tension leading to atomization. When the samples are atomized, fine particles are produced, a small fraction of which then become charged due to microscopic fluctuations in the initial droplet. Recently, SAWN has been combined with other ambient MS ionization techniques such as atmospheric-Pressure chemical Ionization $(\mathrm{APCI})^{140}$ and low-temperature plasma ionization (LTPI) ${ }^{144}$ to enhance the sensitivity. Unlike other microfluidic methods for sample manipulation, SAWN chip does not require pressure driven pumps or interconnects, nor the integration of microchannels. Monkkonen et al. previously combined a DMF device with the SAWN-MS to implement both controllable droplet movement and nebulization processes to perform hydrogen/ deuterium exchange (HDX) of peptides. ${ }^{143,145}$ Recently, the same group integrated anisotropic ratchet conveyors (ARCs) on the SAWN transducer surfaces to automate the sample preparation and droplet delivery process in addition to nebulization. ${ }^{145,146}$ The system did not require the complex control circuitry needed in DMF devices, and the droplet could be manipulated on top of the SAWN chip in an open environment, without an enclosed flow channel or top cover (see Fig. 6). It is important to note that the ion signal from SAWN is typically 2-3 orders of magnitude lower than ESI signal; while SAWN can produce mass spectra similar to ESI, its applications are more limited due to this issue. However, the simple geometry of SAWN devices allows potentially attractive combinations with other ionization techniques to potentially improve overall ionization efficiency. ${ }^{140,144}$

All these recent endeavors of coupling microfluidics with mass spectrometry are summarized in Table 1.

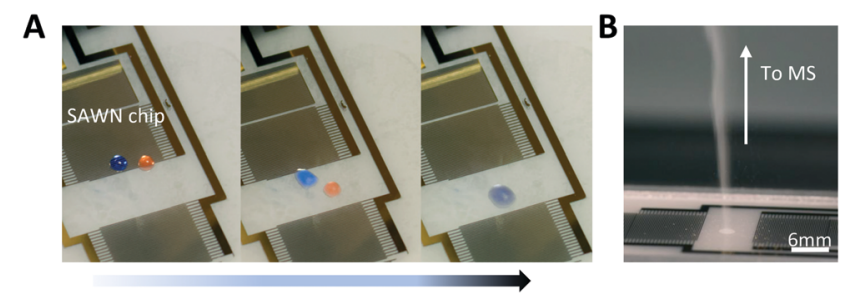

Fig. 6 On-chip surface acoustic wave nebulization (SAWN). (A) Droplet movements (moving, merging, mixing) on the SAWN chip surface integrated with anisotropic ratchet conveyors (ARCs) pattern. ARCs utilize hydrophilic patterns on a hydrophobic background to control the droplet transport by imbalanced hysteresis force. (B) Droplets nebulization. Adapted from ref. 145 with permission from the Royal Society of Chemistry, copyright (C) 2020. 
Table 1 Recent coupling of microfluidics with MS ionization techniques

\begin{tabular}{|c|c|c|c|c|}
\hline \multirow{2}{*}{$\begin{array}{l}\text { Ionization } \\
\text { technique }\end{array}$} & \multirow{2}{*}{$\begin{array}{l}\text { Type of micro- } \\
\text { fluidic system }\end{array}$} & \multirow{2}{*}{ Recent applications [ref.] } & \multicolumn{2}{|l|}{ Microfluidic coupling } \\
\hline & & & Strengths & Challenges \\
\hline \multirow[t]{9}{*}{$\begin{array}{l}\text { Electrospray } \\
\text { ionization (ESI) }\end{array}$} & \multirow[t]{3}{*}{$\begin{array}{l}\text { Continuous } \\
\text { flow }\end{array}$} & $\begin{array}{l}\text { - Single-cell study: in situ lipid } \\
\text { extraction and detection } 160\end{array}$ & $\begin{array}{l}\text { - Ideal for multi-step sample pre- } \\
\text { paration/pretreatment (e.g. Cell lysis, } \\
\text { protein extraction, purification, } \\
\text { separation, concentration) }\end{array}$ & $\begin{array}{l}\text { - Loss of sensitivity due to accumulation } \\
\text { of materials on the ionization source }\end{array}$ \\
\hline & & $\begin{array}{l}\text { - Extraction and quantification of } \\
\text { quinolones in milk }{ }^{192}\end{array}$ & - Coupling with MS is simple & $\begin{array}{l}\text { - Microfluidic chip may require } \\
\text { numerous physical valve structures to } \\
\text { process and ionize multiple samples } \\
\text { in a single device }\end{array}$ \\
\hline & & $\begin{array}{l}\text { - Tumor-endothelial cell } \\
\text { interaction and drug screening }\end{array}$ & $\begin{array}{l}\text { - Flexible adjustment of injection } \\
\text { volume }\end{array}$ & \\
\hline & \multirow[t]{2}{*}{$\begin{array}{l}\text { Microchip } \\
\text { electrophoresis }\end{array}$} & $\begin{array}{l}\text { - Charge variant profiling of } \\
\text { therapeutic proteins }{ }^{164} \\
\text { - Glycoprotein characterization }\end{array}$ & $\begin{array}{l}\text { - Great for sample separation prior to } \\
\text { MS analysis } \\
\text { - Minimal sample required (as low as } \\
\sim 1 \mathrm{~nL} \text { ) }\end{array}$ & $\begin{array}{l}\text { - Use of high voltage during sample } \\
\text { separation/migration } \\
\text { - Sample dilution/loss prior to MS due } \\
\text { to the sheath fluid used in a common } \\
\text { structure }\end{array}$ \\
\hline & & & $\begin{array}{l}\text { - Low flow rate of MCE is compatible } \\
\text { with ESI } \\
\text { - Great for charge variant profiling }\end{array}$ & $\begin{array}{l}\text { - Difficult parallel sample processing } \\
\text { in a single device }\end{array}$ \\
\hline & \multirow[t]{2}{*}{$\begin{array}{l}\text { Droplet } \\
\text { microfluidics }\end{array}$} & $\begin{array}{l}\text { - Analysis of secondary } \\
\text { metabolites from Actinobacteria } \\
\text { - Enzyme activity screening }\end{array}$ & $\begin{array}{l}\text { - Rapid generation and processing of } \\
\text { a large number of sample droplets } \\
\text { - Discrete reaction in each droplet }\end{array}$ & $\begin{array}{l}\text { - Loss of sensitivity due to oil } \\
\text { contamination } \\
\text { - Alternating sample droplet and } \\
\text { carrier oil can interfere with the } \\
\text { formation of a stable ESI plume }\end{array}$ \\
\hline & & $\begin{array}{l}\text { - Detection of pesticide residue } \\
\text { on fruit surfaces }\end{array}$ & $\begin{array}{l}\text { - Minimal sample (pico-to-nanoliter } \\
\text { range) in each droplet }\end{array}$ & $\begin{array}{l}\text { - Potential carryover or cross- } \\
\text { contamination between droplets } \\
\text { - Moving droplets can be influenced } \\
\text { by high ESI potentials (electrowetting) } \\
\text { - Precise droplet manipulation can be } \\
\text { challenging }\end{array}$ \\
\hline & \multirow[t]{2}{*}{$\begin{array}{l}\text { Paper } \\
\text { microfluidics }\end{array}$} & \multirow[t]{2}{*}{$\begin{array}{l}\text { - Urine and hair sample analysis } \\
\text { (Drug abuse screening test) }\end{array}$} & - Low-cost microfluidic device & $\begin{array}{l}\text { - Risk of sample retention and } \\
\text { evaporation during sample delivery } \\
\text { and ionization }\end{array}$ \\
\hline & & & $\begin{array}{l}\text { - Simple device operation (e.g., } \\
\text { simple pipetting) }\end{array}$ & $\begin{array}{l}\text { - Multi-step sample processing is } \\
\text { challenging }\end{array}$ \\
\hline
\end{tabular}

Desorption elec- Continuous trospray ioniza- flow tion (DESI)

\section{Paper} microfluidics
- Analysis of biofluids (e.g., urine, • Little or no sample preparation serum, and cell lysate) ${ }^{10}$

- Quantification of free amino acids in food ${ }^{109}$

- Quantification of targeted miRNAs ${ }^{109}$

- Enzyme activity screening ${ }^{104}$

Matrix-assisted Continuous laser desorption flow ionization

(MALDI) device

- Fast sample preparation using surface
- Analysis of intracellular metabolites ${ }^{114}$

- Separation \& identification of bacteria in food $^{39}$

- Enzyme kinetic assay ${ }^{191}$

- Single-cell metabolic analysis ${ }^{159}$

Droplet microfluidics

- Protein analysis of single yeast cells ${ }^{116}$

- Screening enzyme reaction in yeast cells (secretion/ metabolism) $)^{\mathbf{1 1 5}}$
- Rapid generation of sample in an array format

- A large number of isolated samples can be processed in parallel sample

- Rapid and simple sample preparation sample from sprayed solvents

- Potential cross-contamination of liquid samples in a single device

- Potentially suffer from low efficiency of sample delivery (evaporation, undesired retention) prior to MS analysis

- Low-cost and portable microfluidic • Lower spatial resolution for MS imaging compared to MALDI-MS enclosed flow channel over the MS

- Mostly off-line coupling, requiring manual sample transfer prior to MS analysis

- May require manual separation of MS surface from the microfluidic flow channel

- Potential sample cross-

contamination

- Array generation over the MALDI surface can be challenging

- Matrix coating can compromise sample integrity and cause crosscontamination

- No separation prior to MS lead to high noise if the sample droplet contains a complex biological matrix

- DMF device fabrication is complex - Enzyme activity screening for

Surface-assisted Digital biomass deconstruction ${ }^{133}$ 
Table 1 (continued)

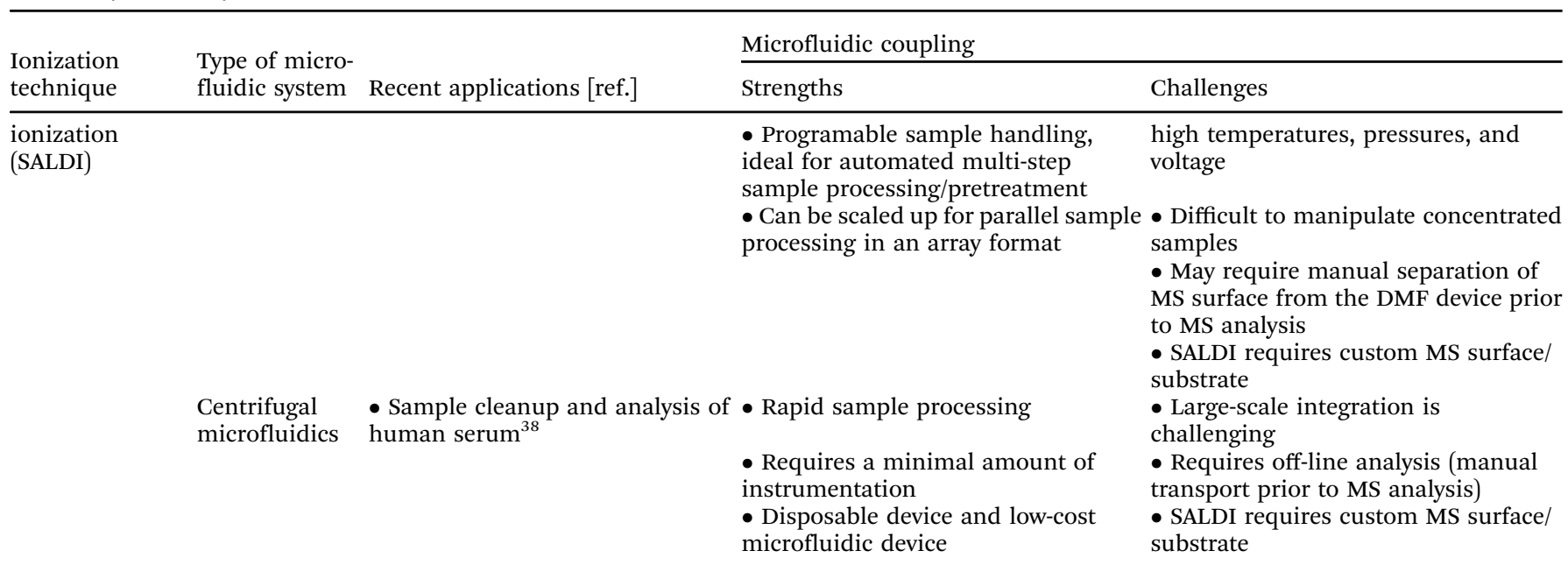

Inductively Droplet $\quad$ Single-cell detection of metal coupled plasma microfluidics ions $^{66,135}$

(ICP)

Acoustic wave nebulization

Surface acous- • Sample droplet manipulation tic

wave

nebulization

(SAWN) chip
- A powerful method to detect trace elements

- Rapid injection and analysis of the • Limited types of sample can be discrete sample in droplets carbon content

analyzed
- Commonly used carrier oil is not ideal for ICP-MS due to relatively high

- Lighter elements (e.g., chromium and iron) are prone to more interferences

- Challenging to manipulate droplets and potential cross-contamination of consecutive droplets

- Compound desorption/ionization • Device fabrication is complex and from the surface without heating the costly sample

- Higher survival yield of fragmentation-prone ions

- Lower ionization efficiency; the ion signal is typically $2-3$ orders of magnitude lower than ESI-MS signal

- Works for both polar and non-polar • Limited to liquid sample (in most analytes cases)

- Difficult to accurately control particle size

\section{Recent applications}

Given the dramatic technical advances in integrating mass spectrometry and microfluidics, a broadening range of applications have been reported in proteomics, metabolomics, cell analysis, and clinical diagnosis. Here we discuss recent applications that have received substantial attention.

\section{A. Single-cell mass spectrometry analysis}

The direct characterization of biomolecules from single cells is an important technical advance. ${ }^{147}$ Technologies that enable the direct measurement of genome, transcriptome, proteome, and metabolome components from individual cells can lead to new insights that would be otherwise unattainable in studies of a bulk population. ${ }^{148}$ Analyzing heterogeneity in cellular responses to chemical and physical perturbations requires miniaturized systems that can perform tens of thousands of experiments on single cells or small communities, and microfluidic approaches have proven to be rapid and cost-effective tools for such research. ${ }^{149}$ Specialized microfluidics systems have been designed for singlecell analysis based on their HTS capabilities, including direct manipulation of cells, controlled cell lysis, and controlled chemical reactions. ${ }^{25,150-152}$ Microfluidics coupled with mass spectrometry has also proven to be a key technology for understanding cellular states, improving upon genome amplification and sequencing approaches, which only yield indirect measurements. Proteomic and metabolomic analyses via mass spectrometry improve detection of cellular states by providing more direct characterization of phenotypes, which is crucial for understanding cellular functions and regulatory networks. ${ }^{147}$

Metabolites are challenging molecules to measure due to their chemical diversity, which encompasses a vast range of concentrations. In order for a mass spectrometry system to reach adequate sensitivity for single-cell metabolomic studies, each step must be optimized. Furthermore, the correct analyte sampling method must be used, and since cell metabolomes rapidly respond to changes in the environment, perturbations upon sampling must be minimized. Over the past few years, different mass spectrometry-based approaches have been developed to profile metabolites in single cells and address these issues. The Sweedler group has pioneered the use of MALDI-MS imaging for studying single-cells by measuring 
chemical variations that can also be used to classify cellular subpopulations. ${ }^{153,154}$ Briefly, cells were dispersed onto a microscope slide before coordinates were assigned via optical imaging; the coordinates were then used to automate MALDI-MS measurements of targeted cells. Such optically guided MALDI-MS works well to assess lipid and peptide content for large populations of cells. Recently, they combined this MALDI-MS imaging with capillary electrophoresis electrospray ionization (CE-ESI-MS) to quantify low-mass metabolites which are difficult to measure because of MALDI matrix interferences. ${ }^{155}$

One strategy developed to increase the throughput of singlecell MALDI-MS measurement involves microarrays for mass spectrometry (MAMS), which feature hydrophilic reservoirs in an omniphobic surface. ${ }^{156,157}$ Applying a cell suspension onto the MAMS surface (which also serves as the MALDI target) enables the rapid and efficient singularization of large numbers of cells in an array format. Such high-density single-cell arrays have been implemented for metabolic analyses of single yeast cells. ${ }^{158}$ More recently, Guillaume-Gentil et al. utilized a high-throughput MAMS surface combined with a new sample collection technique to analyze cytoplasmic metabolites from single live cells. ${ }^{159}$ Unlike previous studies, the intracellular metabolites were analyzed without needing to remove the cell from its environment and cell viability was maintained. Using fluidic force microscopy, quantitative withdrawal of intracellular fluid at sub-picoliter resolution from individually arrayed cells was performed, and the fluids were then automatically transferred to another surface to create an array for MALDI-MS analysis (Fig. 7A). The extraction of $1 \mathrm{pL}$ sample from each cell took around 2-3 min. They demonstrated the detection and identification of 20 metabolites recovered from the cytoplasm of individual HeLa cells while overcoming some of the obstacles often associated with working with live cells. In addition, their approach enabled the analysis of intracellular metabolites at multiple time points and demonstrated possibilities for further investigations of different types of molecules from an individual cell (e.g. combined transcriptional readouts and metabolite profiling).
Another single-cell MS approach was developed to uncover cellular heterogeneity in a human cell line. Huang et al. developed a flow-based microfluidic system for identification and classification of cells, including in-situ extraction of single cells with lipid analysis via an on-line ESI-MS. ${ }^{160}$ Their microfluidics-based in situ single-cell recognition system (ISCRS) can physically isolate single cells using PDMS valve structures and extract phosphatidylcholine for downstream MS analysis (Fig. 7B). The extraction from an isolated cell took $\sim 12$ min. However, the throughput could be increased by adding multiple chambers for parallel sample processing within a single device. This method can be useful for automated multi-step pretreatment of cells.

\section{B. Compound screening}

Compound screening is important in many areas such as drug discovery and bioproduct developments. In particular, by re-engineering an amino acid sequence through directed evolution, enzymes can be developed to explore new substrate and reaction conditions. ${ }^{161,162}$ New compounds produced through this process can be of major biological and chemical importance, but screening enzyme libraries is often the rate-limiting step in drug and biomarker discovery programs. Hence, the creation of technologies for carrying out rapid analysis of enzyme performance is an area of active research interest. Microfluidics-based HTS technology can be an effective option to dramatically decrease the assay volume required and increase the rate of analysis.

Droplet microfluidics interfaced with ESI-MS provides a label-free HTS platform. Diefenbach et al. developed a droplet-MS method in a pharmaceutical setting for industrial enzyme screening, in addition to exploring methods to improve overall throughput of the system. ${ }^{163}$ The enzymatic assays were carried out in a multi-well plate and sample droplets were generated from each well using a custom Teflon fluid path. The droplets were then infused into ESI-MS (Fig. 8A). They demonstrated droplet enzyme reactions using two different transaminase libraries and analyzed the intact reaction mixture
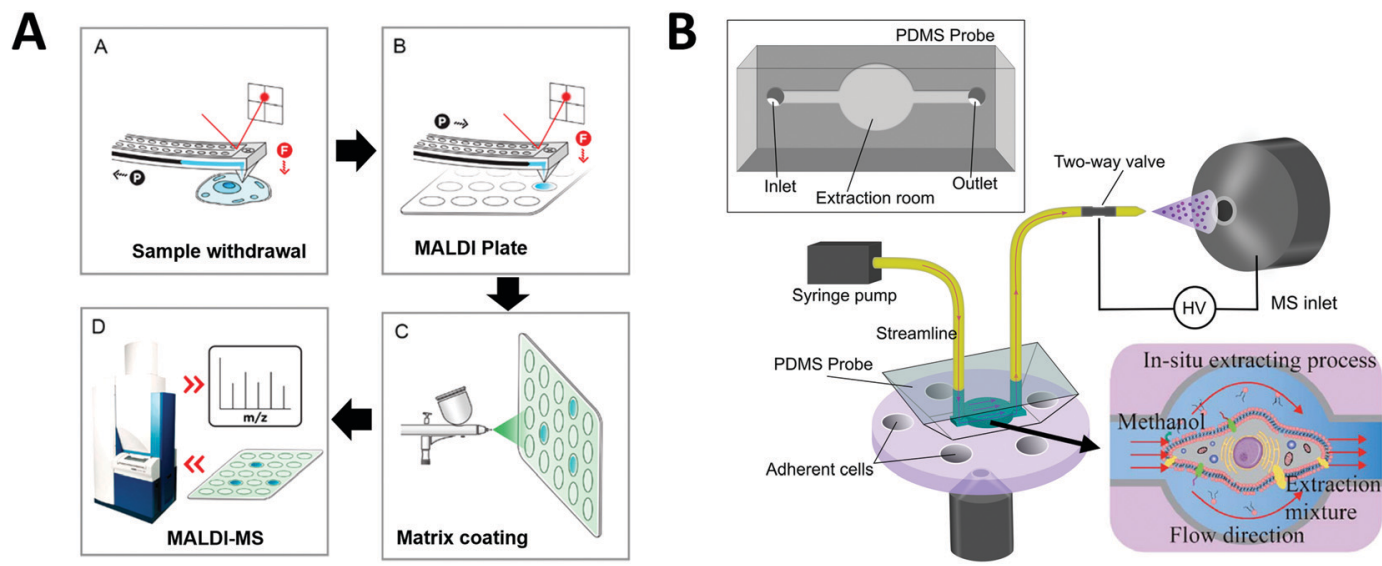

Fig. 7 Applications of microfluidic-mass spectrometry in single-cell analysis. (A) Single-cell metabolite analysis by withdrawing intracellular fluid using fluidic force microscopy, followed by MALDI-MS analysis. Adapted from ref. 159 with permission from the American Chemical Society, copyright $(2017$. (B) Microfluidic in situ single-cell entrapment and extraction for online ESI-MS analysis for single-cell identification and classification. Adapted from ref. 160 with permission from the Royal Society of Chemistry, copyright (C) 2019. 
droplets by ESI-MS. Throughput was improved to $3 \mathrm{~Hz}$ compared to their previous system $(1.7 \mathrm{~Hz})$, with a wide range of droplet sizes (10-50 nL) achieved by tuning the sheath flow within the CE-MS source. These results suggest that mass spectrometry analysis of microfluidic droplets could significantly accelerate processes that require fast throughput, such as the screening of enzyme evolution libraries.

Enzyme reactions carried out in droplets can also be arrayed on discrete SALDI plates. As mentioned in the previous section 'SALDI-MS', Heinemann et al. developed a DMF device patterned with an array of local SALDI-MS surface for HTS of enzyme kinetics at defined time intervals. As a proof-ofconcept, a glycoside hydrolase enzyme (CelECC_CBM3a) was screened against a glycan substrate $(1,4-b$-D-cellotetraose-probe as a model plant biomass) for biofuels and bioenergy applications. ${ }^{133}$ This research demonstrates that such arraybased SALDI-MS approaches have potential for screening large compound libraries and we expect to see developments for other existing SALDI surfaces in the future.

Charge variant profiling of therapeutic proteins is required by the International Council for Harmonization of Technical Requirements for Pharmaceuticals for Human Use (ICH) and is traditionally performed by CE or ion exchange chromatography. Mass spectrometric determination of charge variant profiles acquired from electrophoretic separation is now possible through improvements in coupling microfluidic CE with MS, as well as the introduction of MS-compatible background electrolytes. Carillo et al. developed an MCE system coupled with ESI-MS using a custom "Zipchip" platform. ${ }^{164}$ With their technology, therapeutic monoclonal antibodies rituximab, trastuzumab, and bevacizumab drug products were separated and analyzed for proteoform identification, with an average mass accuracy of $<15 \mathrm{ppm}$. 52 proteoforms were identified for trastuzumab, while rituximab samples indicated the presence of fragments and sialylated $N$-glycans. Khatri et al. also utilized an MCE-MS system for analysis of glycans, glycopeptides and monosaccharides, which require efficient separation methods for characterization of heterogeneous glycoform populations (Fig. 8B). ${ }^{62}$ Thus they demonstrated glycoproteomics analyses (a challenge if using conventional LC-MS) by improving electrophoretic separation followed by MS analysis. The separation of each sample in this MCE-MS system took around 10-20 min.

Affinity purification (AP) is another powerful technology to elucidate protein-protein interactions in cells and tissues. Zhao et al. developed a multifunction microfluidic system including AP coupled to ESI-MS for on-line analysis of seven different quinolones (QNs) in milk samples. Sample extraction, immunoaffinity enrichment, magnetic separation, elution, and ESI-MS were performed sequentially in a single device (Fig. 8C). This system permits automated on-chip immunoaffinity enrichment and accurate MS detection without additional off-line cleanup procedures.

\section{C. Study of microorganisms}

Currently, microorganisms are often identified using 16S rRNA or 18S rRNA gene sequencing. However, because of the incredible complexity of microbial systems, it requires detailed scientific evaluations that yield both chemical and spatial information. $^{165}$ Mass spectrometry analysis can provide chemical information relevant to genomic and transcriptomic data. In recent years, MALDI-MS has emerged as a promising
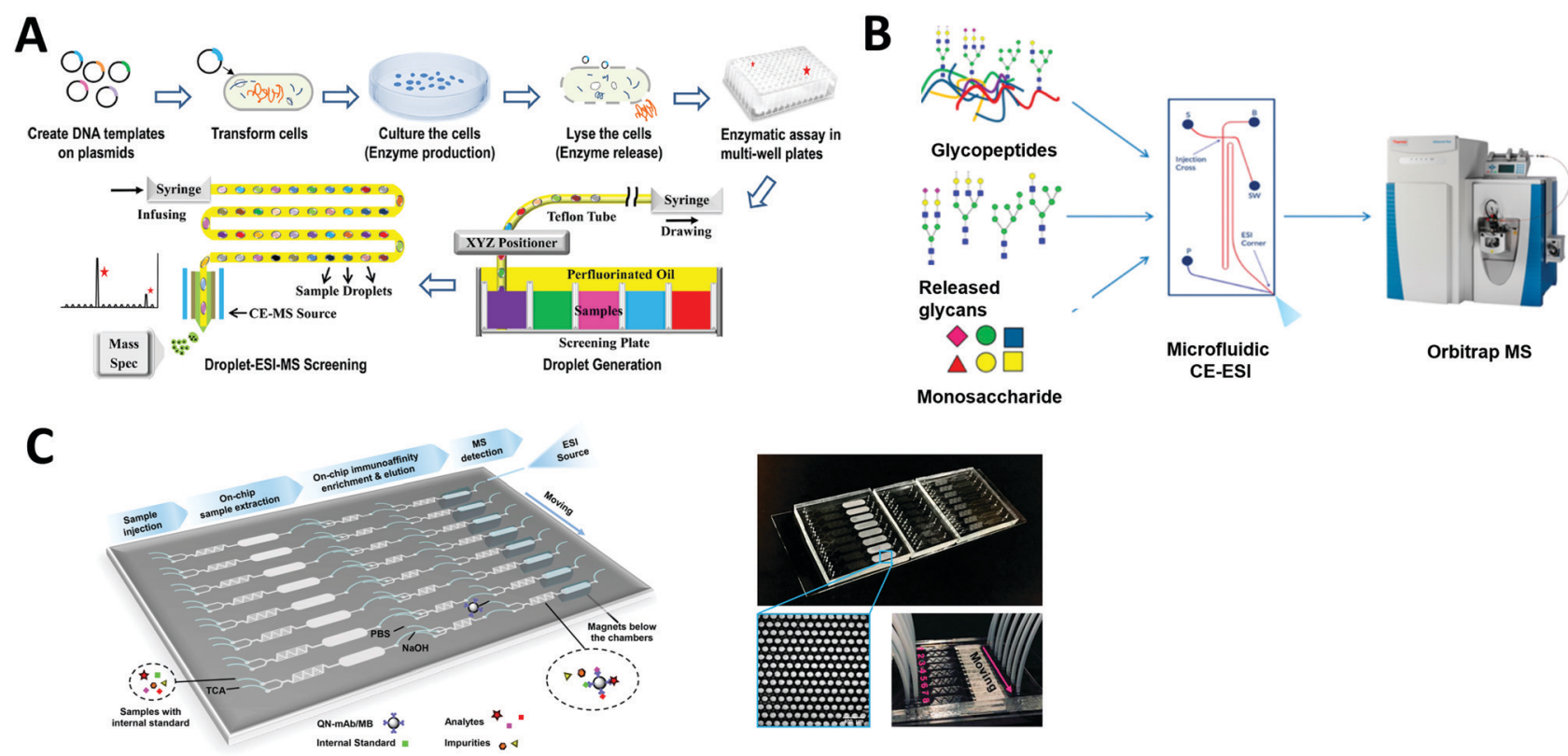

Fig. 8 Applications of microfluidic-mass spectrometry in compound screening. (A) Screening of enzyme libraries using droplet microfluidics interfaced with ESI-MS. Adapted from ref. 163 with permission from the American Chemical Society, copyright (C) 2018. (B) Microfluidic CE-ESI-MS system for analysis of released glycans, glycopeptides and monosaccharides. Adapted from ref. 62 with permission from the American Chemical Society, copyright (C) 2017. (C) Continuous microfluidic device for an automated multi-step sample processing for an online MS analysis of seven different regulated quinolones in milk samples. Adapted from ref. 192 with permission from the American Chemical Society, copyright (C) 2019. 
tool for microbial detection and identification. ${ }^{166-168}$ The Sweedler group, which has pioneered the use of MALDI-MS imaging for studying single-cells, ${ }^{153-155}$ has recently used this approach for high-throughput label-free screening of multistep enzymatic reactions in bacterial colonies. ${ }^{165,169}$ During the MALDI-MS process, microbes are identified using either intact cells or cell extracts and the process is rapid, sensitive, and low cost in terms of labor and reagents. This technology has been widely adapted by microbiologists for bacterial strain typing, including the identification of water- and food-borne pathogens and antibiotic resistance. ${ }^{111}$ However, it is important to note that this technology is limited by peptide mass fingerprint data, as identification of new isolates is dependent on existing database information for a taxonomic rank. For instance, a fingerprint is required when attempting to identify an unknown isolate from a given genera, species, subspecies, or strain.

Condina et al. reported a microfluidic device for separation of microbes, followed by off-line identification of beer spoilage microbes using MALDI-MS. ${ }^{39}$ Their system combines inertial microfluidics with secondary flows in a spiral microchannel for high-throughput separation of yeasts (Saccharomyces pastorianus and Saccharomyces cerevisiae, $\sim 5 \mu \mathrm{m}$ ) from beer spoilage microorganisms (Lactobacillus brevis and Pediococcus damnosus, $\sim 0.3-3 \mu \mathrm{m}$ ). The microorganisms were then identified at the species level using the MALDI-MS off-chip. Even though the MS analysis was performed separately off-chip, this could be modified for on-line MS analysis in the future with a sample transfer system.

Microfluidic cultivation systems coupled with high resolution MS provide single-cell product analysis and quantification capabilities, which can be very useful for microbial studies. Dusney et al. reported an analytical framework that interfaces microfluidic trapping and cultivation of a few bacterial cells (using their previously developed negative dielectrophoresis (nDEP)-based device) with the analysis of their catalytic products by Fourier-transform ion cyclotron resonance mass spectrometry (FT-ICR-MS). ${ }^{170}$ Using the biocatalytic model system of Corynebacterium glutamicum DM 1919 pSenLys cells, they analyzed cells

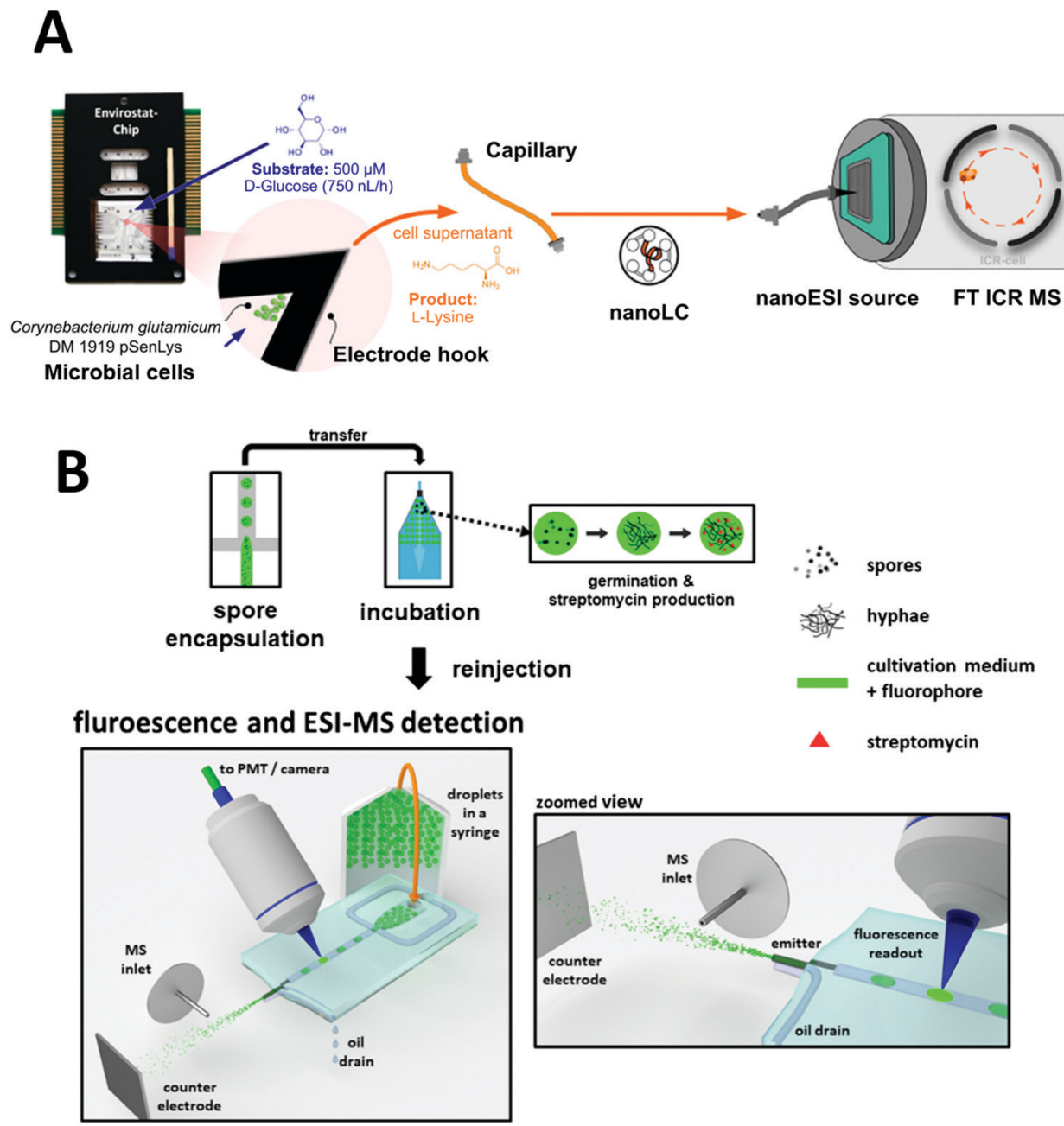

Fig. 9 Applications of microfluidic-mass spectrometry in microbial study. (A) Microfluidic trapping and cultivation of a few microbial cells for the analysis of their catalytic products by Fourier-transform ion cyclotron resonance mass spectrometry. Adapted from ref. 170 with permission from the American Chemical Society, copyright (C) 2019. (B) Encapsulation of spores of Streptomyces griseus in droplets followed by incubation and ESI-MS analysis of produced Streptomycin. Adapted from ref. 99 with permission from Springer Nature, copyright (C) 2018. 
that synthesized L-lysine from D-glucose. Cell trapping (with as few as 19 cells per experiment) was performed on chip for cultivating bacterial cells under continuous perfusion via negative dielectrophoresis (Fig. 9A). Quantification of catalytic products from only a few living cells was also demonstrated via microfluidics coupled with MS; $1.5 \mu \mathrm{L}$ of cell supernatant was sampled into microcapillaries, which was then analyzed using a nanoESI ion source coupled to a FT-ICR-MS. Wink et al. explored a chip that combined MS with an epifluorescence read-out for on-line monitoring of bioactive metabolites produced by incubated Actinobacteria. This was conducted with Streptomyces griseus hyphae encapsulated in droplets of $\sim 200 \mathrm{pL}$. Detection of streptomycin produced in situ via ESI-MS was demonstrated, in addition to highlighting the feasibility of detecting fluorophores inside droplets just before they are electrosprayed (Fig. 9B). ${ }^{99}$ Recently, Terekhov et al. introduced a droplet-generating system equipped with fluorescence-activated cell sorting (FACS) followed by off-chip next-generation sequencing and LC-MS analysis to analyze the secretomes of encapsulated bacteria. ${ }^{23}$

\section{Outlook}

Although enormous strides have been made in microfluidics and their integration with mass spectrometry, further improvements are needed to unlock its full potential as a powerful HTS platform. Here we list several areas that we think are key in this path forward.

\section{Towards on-line chip-to-mass spectrometry coupling}

Real-time in situ measurement of samples by mass spectrometry without any additional handling steps after on-chip operation is ideal. However, the majority of reported approaches still involve off-line sample handling prior MS analysis. Manual steps often include sampling, sample preparation, and sample measurements. These off-chip approaches can compromise sample integrity and lower analysis throughput. Therefore, it will be important to move towards online coupling of microfluidic devices with mass spectrometry analysis for optimal throughput and minimal variation. Additionally, many microfluidic devices will benefit from these developments. For instance, the Abate group has developed a novel technology called 'printed droplet microfluidics', which encapsulates reagents and single cells in picoliter droplets, then actively selects and deposits desired droplets in an arrayed format on a printing substrate. ${ }^{27,64}$ Their systems have been used for various single-cell assays including protein profiling mainly using fluorescence detection and DNA sequencing (and off-chip mass spectrometry analysis in some cases) as readouts. Such an advanced microfluidic platform can take advantage of on-line coupling with mass spectrometry to further expand their applications.

\section{Universal sample processing system}

A major advantage of microfluidic chips is the ability to automate and multiplex multiple processes with various functionalities. It can include cell culture, sample extraction, purification, desalting, concentration, drying, and droplet encapsulation. ${ }^{44,45,171-174}$ More efforts can be made to integrate multiple sample processing/pretreatment steps for biological samples into a single microfluidic system prior to ionization. And this universal sample processing system could be coupled with various types of ionization technique (e.g., ESI, MALDI) for downstream mass analysis. We anticipate, for example, microfluidic devices that can transform cells, culture cells and extract metabolites from the cells to study the gene function at large scale. Such universal sample processing system can be very powerful, introducing the needed experimental flexibility to perform.

\section{Coupling emerging ionization techniques with microfluidics}

In addition to aforementioned ionization techniques, other emerging ionization approaches have been recently coupled with microfluidic devices. For example, Sathyanarayanan et al. developed a digital microfluidic devices equipped with desorption atmospheric pressure photoionization (DAPPI) for the ambient ionization of samples. ${ }^{175}$ This technology uses a microfabricated nebulizer chip with an internal heater to localize the gas jet $\left(250-350{ }^{\circ} \mathrm{C}\right)$ onto the sample droplet, resulting in thermal desorption via gas-phase chemical reactions.

Recently, Sinclair et al. reported an acoustic mist ionization (AMI) for direct injection of charged femto-liter sample droplets into the MS system. ${ }^{176}$ This platform uses acoustic energy (thereby eliminating the need for physical contact) to load ionized liquid samples from a microtiter plate to a mass spectrometer. In AMI-MS, sample ionization is achieved by applying high voltage $(0.5-4 \mathrm{kV})$ above the test well, which leads to charge separation in the sample prior to droplet generation. Then, ultrasonic pulses are delivered from an acoustic transducer to produce a fluid cone and a spray of charged droplets. AMI-MS systems can deliver quantitative results up to 50 times faster than conventional LC-MS, with the system capable of analyzing up to 3 samples per second and 100000 samples per day on a single mass spectrometer. ${ }^{176,177}$ A few commercial AMI-MS (or Echo-MS) platforms have been developed (e.g., XEVO G2-XS QTOF-MS with AMI by Labcyte Inc. \& Waters Corp., Echo ${ }^{\circledR}$ MS by AB SCIEX Ltd). ${ }^{177,178}$ To the best of our knowledge, there have been no publications on microfluidic devices integrated with the AMI-MS. Due to the potential of such technology, the development of microfluidic systems with direct acoustic sample droplet injection methods would be of considerable significance.

\section{Coupling microfluidics with a miniaturized mass spectrometer}

Even with the coupling of mass spectrometer with various microfluidic chips, conventional mass spectrometry system still suffers from portability issue due to its bulky equipment size. ${ }^{179,180}$ Cooks et al. pioneered the miniaturization of mass spectrometry system, called miniature mass spectrometer (MMS). The group has developed multiple generations of MMS (including the $25 \mathrm{~kg}$ "backpack" version) equipped with 
the miniaturized ion trap and ambient ionization source ${ }^{181-183}$ that can perform direct mass spectrometry analysis on complex samples without sample preparation or chromatographic separation (including sample types such as whole blood, untreated food, and environmental samples). Kirby et al. coupled digital microfluidics with the MMS for quantitation of drugs in urine. ${ }^{75} \mathrm{~A}$ custom digital microfluidic system was used to deliver droplets of solvent to dried urine samples, which were analyzed on MMS after the analytes were sent through an array of nanoelectrospray emitters. Cocaine, benzoylecgonine, and codeine were quantified in less than $15 \mathrm{~min}$ from four dried urine samples.

To make microfluidic-mass spectrometry systems truly portable, more coupling of various microfluidic systems with improved MMS can be explored.

\section{Higher specificity}

Separation of samples prior to mass spectrometry analysis is important to improve sensitivity and specificity, especially if the analytes of interests are in complex biological matrices. In addition to on-chip purification or separation capabilities via microchip electrophoresis or microchip liquid chromatography (separation column embedded on chip), ${ }^{82,83}$ other off-chip separation techniques such as ion-mobility spectrometry (IMS) can be coupled with mass spectrometry to further enhance detection specificity of the microfluidic-mass spectrometry system. IMS separates ions based on the difference in mobility in an electric field in the gas phase, caused by their mass, shape/ size and charge and integration with mass spectrometry can result in rapid analyte separation for mass spectrometry-based measurements. ${ }^{184}$ An increasing number of commercial IMS-MS platforms from several different vendors are available. ${ }^{185}$ This combined with microfluidic sample preparation will allow for further improvement in specificity. Another approach to improve MS detection specificity is tandem mass spectrometry (MS/MS). MS/MS involves two stages, where in the first stage ions of a desired $\mathrm{m} / \mathrm{z}$ are isolated from the rest of the ions emanating from the ion source. These precursor ions are then induced to undergo a process to increase the internal energy of the ions leading to fragmentation. The ions resulting from the reactions are termed product ions, and these are analyzed with the second stage of MS/MS. The resulting MS/MS spectra provide additional specificity and can be compared with reference MS/MS spectra to further support chemical identifications.

\section{Multi-omics platforms}

Genomics and transcriptomics though DNA and RNA sequencing have emerged as powerful tools for characterizing cells, including single cells. However, not all phenotypes of interest can be observed through changes in gene expression. For example, traditional sequencing approaches cannot capture the epigenetic state, protein expression, enzyme activity, and morphology of a cell or set of cells. ${ }^{65}$ Thus, combining genomic/transcriptomic analyses with proteomic and metabolomic analyses via mass spectrometry into a single microfluidic system could reveal genotype-to-phenotype relationships ${ }^{23}$ and provide insight into the molecular basis of cellular function. ${ }^{23}$ As a recent example, Zhang et al. reported a droplet microfluidic platform to connect optical imaging with gene expression profiling of single cells. ${ }^{65}$ In the future, such system could be also coupled with MS for more comprehensive characterization of single cells.

A range of other analytical techniques can also be coupled with microfluidics and mass spectrometry such as surfaceplasmon resonance (SPR) and surface enhanced Raman spectroscopy (SERS). SPR sensors can provide quantitative real-time binding data particularly useful to investigate the protein-protein interactions. ${ }^{19}$ However, as the monitoring of an interaction between a protein or small molecular ligands and a receptor molecule provides ambiguous information on the identity of the bound material due to lack of selectivity, a second technique is necessary for identification. ${ }^{186}$ The combination of SPR and mass spectrometry is emerging as a sensitive tool for the elucidation of novel protein-protein interactions as recently reported. ${ }^{14,186,187}$ Another powerful analytical technique, SERS detects Raman signal enhancement of analytes located close to or directly adsorbed onto the metal (nanoparticle) surface. ${ }^{17,20}$ There have been efforts of integrating SERS with mass spectrometry including the recent example of coupling SERS with paper spray ionizatio. ${ }^{15,16,188}$ These coupling approaches can, for example, enable analysis of cell biomass which could facilitate normalization of mass spectrometry data.

\section{Detection of pathogenic microorganisms}

The recent appearance of the novel corona virus (SARS-CoV-2 or COVID-19) and its alarming spread highlighted the importance of rapid diagnostic systems. ${ }^{189}$ Currently, laboratory-based reverse transcription-polymerase chain reaction (RT-PCR) is the primary method of confirming COVID-19 infection. However, RT-PCR tests demand well-equipped laboratories and skilled personnel. To address this challenging situation, rapid testing is under development, with a large component based on later-flow microfluidics (or $\mu$ PADs) to make diagnostic devices faster and more affordable. ${ }^{81}$ In addition to common colorimetric readouts, paper microfluidics could accurately identify and quantify analytes of interest via downstream MS analysis, such as LDI-MS or L-DESI. Recently, Nachtigall et al. has proposed to use MALDI-MS pathogen identification combined with machine learning analysis for SARS-CoV-2 testing. ${ }^{112}$ They obtained mass spectra from 362 samples and, through the use of machine learning, were able to analyze and select the peaks that could distinguish a positive SARS-CoV-2 sample from a negative one. Such approaches can potentially become powerful diagnostic tools if sample processing can be performed via microfluidics. Based on recent advances in microfluidics and its coupling with MS techniques, microfluidic-MS would enable rapid and accurate detection of various pathogenic microorganisms. If further developed and properly validated such new approaches would enable point-ofcare testing, leading to more efficient and decentralized screening among suspected cases. 


\section{Conclusions}

Advanced microfluidics enables the translation of chemical and biological assays to scales and rates unachievable in conventional laboratory workflows. The successful integration of microfluidic systems with mass spectrometry analysis provides a powerful approach to increase the sensitivity, specificity, and throughput of many conventional assays. It also allows for the integration and automation of sample processing to minimize sample and reagent consumption and to further streamline experimental workflows to make faster, better, and cheaper assays. Currently, a wide variety of mass spectrometry ionization techniques have been effectively coupled to different microfluidic systems and used for diverse applications. We expect that the integration of additional mass spectrometry capabilities outside the microfluidic chips such as the Ionmobility spectrometry-mass spectrometry (IMS-MS) or tandem MS/MS configuration can also further enhance the specificity of these assays. Despite substantial advancements in microfluidic sample manipulation and analysis, there are still challenges for many microfluidic devices to be widely adapted for highthroughput screening applications. Although we believe that all systems reviewed here have the potential for increased throughput via various methods, some technologies have not yet been demonstrated for high-throughput assays. Broad adoption of these techniques will require robust implementation of strategies for the stable sample storage, containment, and sample tracking. Overall, we are optimistic that microfluidic-mass spectrometry systems will provide faster analysis while offering more sensitive and comprehensive analyses that eliminate off-line or manual sample handling steps to enable massive-scale screening capabilities.

\section{Conflicts of interest}

There are no conflicts to declare.

\section{Acknowledgements}

Contributions from $\mathrm{NH}$ and $\mathrm{CP}$ were funded by the DOE Joint BioEnergy Institute (http://www.jbei.org) and those of MdR, LZH, and AG were funded by the ENIGMA-Ecosystems and Networks Integrated with Genes and Molecular Assemblies (http://enigma.lbl.gov) Science Focus Area Program, and TN was funded by both. Both JBEI and ENIGMA are supported by the U.S. Department of Energy, Office of Science, Office of Biological and Environmental Research, through contract DE-AC02-05CH11231 between Lawrence Berkeley National Laboratory and the U.S. Department of Energy. The United States Government retains and the publisher, by accepting the article for publication, acknowledges that the United States Government retains a non-exclusive, paid-up, irrevocable, world-wide license to publish or reproduce the published form of this manuscript, or allow others to do so, for United States Government purposes.

\section{References}

1 S. Fox, S. Farr-Jones, L. Sopchak, A. Boggs, H. W. Nicely, R. Khoury and M. Biros, J. Biomol. Screening, 2006, 11(7), 864-869.

2 R. Macarron, M. N. Banks, D. Bojanic, D. J. Burns, D. A. Cirovic, T. Garyantes, D. V. S. Green, R. P. Hertzberg, W. P. Janzen, J. W. Paslay, U. Schopfer and G. S. Sittampalam, Nat. Rev. Drug Discovery, 2011, 10, 188-195.

3 E. E. Kempa, K. A. Hollywood, C. A. Smith and P. E. Barran, Analyst, 2019, 144, 872-891.

4 L. M. Mayr and D. Bojanic, Curr. Opin. Pharmacol., 2009, 9, 580-588.

5 S. A. Sundberg, Curr. Opin. Biotechnol, 2000, 11, 47-53.

6 E. M. Payne, D. A. Holland-Moritz, S. Sun and R. T. Kennedy, Lab Chip, 2020, 20, 2247-2262.

7 A. El-Aneed, A. Cohen and J. Banoub, Appl. Spectrosc. Rev., 2009, 44, 210-230.

8 T. P. Roddy, C. R. Horvath, S. J. Stout, K. L. Kenney, P.-I. Ho, J.-H. Zhang, C. Vickers, V. Kaushik, B. Hubbard and Y. K. Wang, Anal. Chem., 2007, 79, 8207-8213.

9 M. Bantscheff, M. Schirle, G. Sweetman, J. Rick and B. Kuster, Anal. Bioanal. Chem., 2007, 389, 1017-1031.

10 M. de Raad, C. R. Fischer and T. R. Northen, Curr. Opin. Chem. Biol., 2016, 30, 7-13.

11 D. B. Liesenfeld, N. Habermann, R. W. Owen, A. Scalbert and C. M. Ulrich, Cancer Epidemiol., Biomarkers Prev., 2013, 22, 2182-2201.

12 K. Deng, J. M. Guenther, J. Gao, B. P. Bowen, H. Tran, V. Reyes-Ortiz, X. Cheng, N. Sathitsuksanoh, R. Heins, T. E. Takasuka, L. F. Bergeman, H. Geertz-Hansen, S. Deutsch, D. Loqué, K. L. Sale, B. A. Simmons, P. D. Adams, A. K. Singh, B. G. Fox and T. R. Northen, Front. Bioeng. Biotechnol., 2015, 3, 153.

13 N. F. C. Visser, A. Scholten, R. H. H. van den Heuvel and A. J. R. Heck, ChemBioChem, 2007, 8, 298-305.

14 A. Madeira, E. Öhman, A. Nilsson, B. Sjögren, P. E. Andrén and P. Svenningsson, Nat. Protoc., 2009, 4, 1023-1037.

15 B. Nie, R. N. Masyuko and P. W. Bohn, Analyst, 2012, 137, 1421-1427.

16 G. Grasso, L. D’Urso, E. Messina, F. Cataldo, O. Puglisi, G. Spoto and G. Compagnini, Carbon, 2009, 47, 2611-2619.

17 J. Langer, D. Jimenez de Aberasturi, J. Aizpurua, R. A. Alvarez-Puebla, B. Auguié, J. J. Baumberg, G. C. Bazan, S. E. J. Bell, A. Boisen, A. G. Brolo, J. Choo, D. Cialla-May, V. Deckert, L. Fabris, K. Faulds, F. J. García de Abajo, R. Goodacre, D. Graham, A. J. Haes, C. L. Haynes, C. Huck, T. Itoh, M. Käll, J. Kneipp, N. A. Kotov, H. Kuang, E. C. Le Ru, H. K. Lee, J.-F. Li, X. Y. Ling, S. A. Maier, T. Mayerhöfer, M. Moskovits, K. Murakoshi, J.-M. Nam, S. Nie, Y. Ozaki, I. Pastoriza-Santos, J. Perez-Juste, J. Popp, A. Pucci, S. Reich, B. Ren, G. C. Schatz, T. Shegai, S. Schlücker, L.-L. Tay, K. G. Thomas, Z.-Q. Tian, R. P. Van Duyne, T. Vo-Dinh, Y. Wang, K. A. Willets, 
C. Xu, H. Xu, Y. Xu, Y. S. Yamamoto, B. Zhao and L. M. Liz-Marzán, ACS Nano, 2020, 14, 28-117.

18 I. Abdulhalim, M. Zourob and A. Lakhtakia, Electromagnetics, 2008, 28, 214-242.

19 V. Amendola, R. Pilot, M. Frasconi, O. M. Maragò and M. A. Iatì, J. Phys.: Condens. Matter, 2017, 29, 203002.

20 S. Fornasaro, F. Alsamad, M. Baia, L. A. E. Batista de Carvalho, C. Beleites, H. J. Byrne, A. Chiadò, M. Chis, M. Chisanga, A. Daniel, J. Dybas, G. Eppe, G. Falgayrac, K. Faulds, H. Gebavi, F. Giorgis, R. Goodacre, D. Graham, P. La Manna, S. Laing, L. Litti, F. M. Lyng, K. Malek, C. Malherbe, M. P. M. Marques, M. Meneghetti, E. Mitri, V. Mohaček-Grošev, C. Morasso, H. Muhamadali, P. Musto, C. Novara, M. Pannico, G. Penel, O. Piot, T. Rindzevicius, E. A. Rusu, M. S. Schmidt, V. Sergo, G. D. Sockalingum, V. Untereiner, R. Vanna, E. Wiercigroch and A. Bonifacio, Anal. Chem., 2020, 92, 4053-4064.

21 H. Kim, M. J. Jebrail, A. Sinha, Z. W. Bent, O. D. Solberg, K. P. Williams, S. A. Langevin, R. F. Renzi, J. L. Van De Vreugde, R. J. Meagher, J. S. Schoeniger, T. W. Lane, S. S. Branda, M. S. Bartsch and K. D. Patel, PLoS One, 2013, 8, e68988.

22 T. W. Murphy, Y.-P. Hsieh, B. Zhu, L. B. Naler and C. Lu, Anal. Chem., 2020, 92, 2519-2526.

23 S. S. Terekhov, I. V. Smirnov, A. V. Stepanova, T. V. Bobik, Y. A. Mokrushina, N. A. Ponomarenko, A. A. Belogurov, M. P. Rubtsova, O. V. Kartseva, M. O. Gomzikova, A. A. Moskovtsev, A. S. Bukatin, M. V. Dubina, E. S. Kostryukova, V. V. Babenko, M. T. Vakhitova, A. I. Manolov, M. V. Malakhova, M. A. Kornienko, A. V. Tyakht, A. A. Vanyushkina, E. N. Ilina, P. Masson, A. G. Gabibov and S. Altman, Proc. Natl. Acad. Sci. U. S. A., 2017, 114, 2550-2555.

24 S. R. Heron, R. Wilson, S. A. Shaffer, D. R. Goodlett and J. M. Cooper, Anal. Chem., 2010, 82, 3985-3989.

25 H. N. Joensson and H. Andersson Svahn, Angew. Chem., Int. Ed., 2018, 12176-12192.

26 M. T. Guo, A. Rotem, J. A. Heyman and D. A. Weitz, Lab Chip, 2012, 12, 2146-2155.

27 R. H. Cole, S.-Y. Tang, C. A. Siltanen, P. Shahi, J. Q. Zhang, S. Poust, Z. J. Gartner and A. R. Abate, Proc. Natl. Acad. Sci. U. S. A., 2017, 114, 8728-8733.

28 R. Seemann, M. Brinkmann, T. Pfohl and S. Herminghaus, Rep. Prog. Phys., 2012, 75, 016601.

29 A. Kulesa, J. Kehe, J. E. Hurtado, P. Tawde and P. C. Blainey, Proc. Natl. Acad. Sci. U. S. A., 2018, 115, 6685-6690.

30 N. S. Ha, S. Sadeghi and R. M. Van Dam, Micromachines, 2017, 8, 337.

31 D. B. Weibel and G. M. Whitesides, Curr. Opin. Chem. Biol., 2006, 10, 584-591.

32 D. Gao, C. Song and J.-M. Lin, in Microfluidics for SingleCell Analysis, ed. J.-M. Lin, Springer, Singapore, 2019, pp. 163-195.

33 X. Wang, L. Yi, N. Mukhitov, A. M. Schrell, R. Dhumpa and M. G. Roper, J. Chromatogr. A, 2015, 1382, 98-116.
34 P. S. Dittrich, K. Tachikawa and A. Manz, Anal. Chem., 2006, 78, 3887-3908.

35 E. A. Redman, J. S. Mellors, J. A. Starkey and J. M. Ramsey, Anal. Chem., 2016, 88, 2220-2226.

36 T. M. Squires and S. R. Quake, Rev. Mod. Phys., 2005, 77, 977-1026.

37 J. Melin and S. R. Quake, Annu. Rev. Biophys. Biomol. Struct., 2007, 36, 213-231.

38 Y. Zhao, Y. Hou, J. Ji, F. Khan, T. Thundat and D. J. Harrison, Anal. Chem., 2019, 91, 7570-7577.

39 M. R. Condina, B. A. Dilmetz, S. R. Bazaz, J. Meneses, M. E. Warkiani and P. Hoffmann, Lab Chip, 2019, 19, 1961-1970.

40 T. Ngernsutivorakul, D. J. Steyer, A. C. Valenta and R. T. Kennedy, Anal. Chem., 2018, 90, 10943-10950.

41 T. Thorsen, S. J. Maerkl and S. R. Quake, Science, 2002, 298, 580-584.

42 F. K. Balagaddé, L. You, C. L. Hansen, F. H. Arnold and S. R. Quake, Science, 2005, 309, 137-140.

43 N. Gasilova, K. Srzentić, L. Qiao, B. Liu, A. Beck, Y. O. Tsybin and H. H. Girault, Anal. Chem., 2016, 88, 1775-1784.

44 L. Lin, X. Lin, L. Lin, Q. Feng, T. Kitamori, J.-M. Lin and J. Sun, Anal. Chem., 2017, 89, 10037-10044.

45 S. Kumar, V. Sahore, C. I. Rogers and A. T. Woolley, Analyst, 2016, 141, 1660-1668.

46 X. Sun, W. Yang, Y. Geng and A. T. Woolley, Lab Chip, 2009, 9, 949-953.

47 J. A. Jankowski, S. Tracht and J. V. Sweedler, TrAC, Trends Anal. Chem., 1995, 14, 170-176.

48 N. S. Ha, J. Ly, J. Jones, S. Cheung and R. M. van Dam, Anal. Chim. Acta, 2017, 985, 129-140.

49 G. G. Mironov, C. M. Clouthier, A. Akbar, J. W. Keillor and M. V. Berezovski, Nat. Chem. Biol., 2016, 12, 918-922.

50 G. G. Morbioli, T. Mazzu-Nascimento, A. Aquino, C. Cervantes and E. Carrilho, Anal. Chim. Acta, 2016, 935, 44-57.

51 B. C. Durney, C. L. Crihfield and L. A. Holland, Anal. Bioanal. Chem., 2015, 407, 6923-6938.

52 Z. Yang and J. V. Sweedler, Anal. Bioanal. Chem., 2014, 406, 4013-4031.

53 A. N. de Macedo, M. Irfan Yasin Jiwa, J. Macri, V. Belostotsky, S. Hill and P. Britz-McKibbin, Anal. Chem., 2013, 85, 11112-11120.

54 S.-K. Ruokonen, F. Duša, J. Lokajová, I. Kilpeläinen, A. W. T. King and S. K. Wiedmer, J. Chromatogr. A, 2015, 1405, 178-187.

55 A. M. Skelley, J. R. Scherer, A. D. Aubrey, W. H. Grover, R. H. C. Ivester, P. Ehrenfreund, F. J. Grunthaner, J. L. Bada and R. A. Mathies, Proc. Natl. Acad. Sci. U. S. A., 2005, 102, 1041-1046.

56 E. R. Castro and A. Manz, J. Chromatogr. A, 2015, 1382, 66-85.

57 D. Janasek, J. Franzke and A. Manz, Nature, 2006, 442, 374-380. 
58 J. Jones, N. S. Ha, A. G. Barajas, A. F. Chatziioannou and R. M. van Dam, Anal. Chem., 2020, 92(4), 3483-3491.

59 J. Ly, N. S. Ha, S. Cheung and R. M. van Dam, Anal. Bioanal. Chem., 2018, 410, 2423-2436.

60 N. G. Batz, J. S. Mellors, J. P. Alarie and J. M. Ramsey, Anal. Chem., 2014, 86, 3493-3500.

61 A. Pettersson Dahlin, M. Wetterhall, G. Liljegren, S. K. Bergström, P. Andrén, L. Nyholm, K. E. Markides and J. Bergquist, Analyst, 2005, 130, 193-199.

62 K. Khatri, J. A. Klein, J. R. Haserick, D. R. Leon, C. E. Costello, M. E. McComb and J. Zaia, Anal. Chem., 2017, 89, 6645-6655.

63 D. T. Chiu, R. M. Lorenz and G. D. M. Jeffries, Anal. Chem., 2009, 81, 5111-5118.

64 P. Shahi, S. C. Kim, J. R. Haliburton, Z. J. Gartner and A. R. Abate, Sci. Rep., 2017, 7, 1-12.

65 J. Q. Zhang, C. A. Siltanen, L. Liu, K.-C. Chang, Z. J. Gartner and A. R. Abate, Genome Biol., 2020, 21, 49.

66 Y. Zhou, Z. Chen, J. Zeng, J. Zhang, D. Yu, B. Zhang, X. Yan, L. Yang and Q. Wang, Anal. Chem., 2020, 92, 5286-5293.

67 R. T. Kelly, J. S. Page, I. Marginean, K. Tang and R. D. Smith, Angew. Chem., Int. Ed., 2009, 48, 6832-6835.

68 X. Sun, K. Tang, R. D. Smith and R. T. Kelly, Microfluid. Nanofluid., 2013, 15, 117-126.

69 Y. Zhu and Q. Fang, Anal. Chem., 2010, 82, 8361-8366.

70 K. Choi, A. H. C. Ng, R. Fobel and A. R. Wheeler, Annu. Rev. Anal. Chem., 2012, 5, 413-440.

71 I. Barbulovic-Nad, S. H. Au and A. R. Wheeler, Lab Chip, 2010, 10, 1536-1542.

72 P. Y. Keng, S. Chen, H. Ding, S. Sadeghi, G. J. Shah, A. Dooraghi, M. E. Phelps, N. Satyamurthy, A. F. Chatziioannou, C.-J. 'CJ' Kim and R. M. van Dam, Proc. Natl. Acad. Sci. U. S. A., 2012, 109, 690-695.

73 J. Li, N. S. Ha, T. Leo' Liu, R. M. van Dam and C.-J. 'CJ' Kim, Nature, 2019, 572, 507-510.

74 A. R. Wheeler, H. Moon, C.-J. Kim, J. A. Loo and R. L. Garrell, Anal. Chem., 2004, 76, 4833-4838.

75 A. E. Kirby and A. R. Wheeler, Anal. Chem., 2013, 85, 6178-6184.

76 M. Tang, G. Wang, S.-K. Kong and H.-P. Ho, Micromachines, 2016, 7, 26.

77 T.-H. Kim, K. Abi-Samra, V. Sunkara, D.-K. Park, M. Amasia, N. Kim, J. Kim, H. Kim, M. Madou and Y.-K. Cho, Lab Chip, 2013, 13, 3747-3754.

78 R. Gorkin, J. Park, J. Siegrist, M. Amasia, B. Seok Lee, J.-M. Park, J. Kim, H. Kim, M. Madou and Y.-K. Cho, Lab Chip, 2010, 10, 1758-1773.

79 A. W. Martinez, S. T. Phillips and G. M. Whitesides, Proc. Natl. Acad. Sci. U. S. A., 2008, 105, 19606-19611.

80 X. Li, D. R. Ballerini and W. Shen, Biomicrofluidics, 2012, 6, 011301.

81 A. W. Martinez, S. T. Phillips, G. M. Whitesides and E. Carrilho, Anal. Chem., 2010, 82, 3-10.

82 I. M. Lazar, P. Trisiripisal and H. A. Sarvaiya, Anal. Chem., 2006, 78, 5513-5524.
83 R. F. Gerhardt, A. J. Peretzki, S. K. Piendl and D. Belder, Anal. Chem., 2017, 89, 13030-13037.

84 J. Stadlmann, O. Hudecz, G. Krššáková, C. Ctortecka, G. Van Raemdonck, J. Op De Beeck, G. Desmet, J. M. Penninger, P. Jacobs and K. Mechtler, Anal. Chem., 2019, 91, 14203-14207.

85 J. O. de Beeck and F. Impens, A Novel Nanoflow LC-MS Approach for Bottom-up Proteomics Using Micro Pillar Array Columns, PharmaFluidics, 2018, https://www.pharma fluidics.com/products/\%c2\%b5pac-caplc-column/.

86 C. A. Croushore, S. Supharoek, C. Y. Lee, J. Jakmunee and J. V. Sweedler, Anal. Chem., 2012, 84, 9446-9452.

87 T. Scholl, C. Dietze, M. Schmidt, S. Ohla and D. Belder, Anal. Bioanal. Chem., 2018, 410, 5741-5750.

88 R. T. Kelly, K. Tang, D. Irimia, M. Toner and R. D. Smith, Anal. Chem., 2008, 80, 3824-3831.

89 X. Sun, R. T. Kelly, K. Tang and R. D. Smith, Analyst, 2010, 135, 2296-2302.

90 R. T. Kelly, C. Wang, S. J. Rausch, C. S. Lee and K. Tang, Anal. Chem., 2014, 86, 6723-6729.

91 Y. Cong, S. Katipamula, C. D. Trader, D. J. Orton, T. Geng, E. S. Baker and R. T. Kelly, Lab Chip, 2016, 16, 1544-1548.

92 S. Sun and R. T. Kennedy, Anal. Chem., 2014, 86, 9309-9314.

93 D. J. Steyer and R. T. Kennedy, Anal. Chem., 2019, 91, 6645-6651.

94 A. J. Peretzki, S. Schmidt, E. Flachowsky, A. Das, R. F. Gerhardt and D. Belder, Lab Chip, 2020, 20, 4456-4465.

95 C.-M. Huang, Y. Zhu, D.-Q. Jin, R. T. Kelly and Q. Fang, Anal. Chem., 2017, 89, 9009-9016.

96 J. Liu, H. Wang, N. E. Manicke, J.-M. Lin, R. G. Cooks and Z. Ouyang, Anal. Chem., 2010, 82, 2463-2471.

97 G. I. J. Salentijn, H. P. Permentier and E. Verpoorte, Anal. Chem., 2014, 86, 11657-11665.

98 Y.-C. Li, M.-H. Cheng and C.-H. Lin, in 2020 IEEE 33rd International Conference on Micro Electro Mechanical Systems (MEMS), 2020, pp. 98-101.

99 K. Wink, L. Mahler, J. R. Beulig, S. K. Piendl, M. Roth and D. Belder, Anal. Bioanal. Chem., 2018, 410, 7679-7687.

100 C. A. Smith, X. Li, T. H. Mize, T. D. Sharpe, E. I. Graziani, C. Abell and W. T. S. Huck, Anal. Chem., 2013, 85, 3812-3816.

101 C. S. Clendinen, M. E. Monge and F. M. Fernández, Analyst, 2017, 142, 3101-3117.

102 G. A. Harris, A. S. Galhena and F. M. Fernández, Anal. Chem., 2011, 83, 4508-4538.

103 M. Wleklinski, B. P. Loren, C. R. Ferreira, Z. Jaman, L. Avramova, T. J. P. Sobreira, D. H. Thompson and R. G. Cooks, Chem. Sci., 2018, 9, 1647-1653.

104 S. V. de Freitas, F. R. de Souza, J. C. R. Neto, G. A. Vasconcelos, P. V. Abdelnur, B. G. Vaz, C. S. Henry and W. K. T. Coltro, Anal. Chem., 2018, 90(20), 11949-11954.

105 E. Gemperline, B. Chen and L. Li, Bioanalysis, 2014, 6, 525-540. 
106 J. Laskin, B. S. Heath, P. J. Roach, L. Cazares and O. J. Semmes, Anal. Chem., 2012, 84, 141-148.

107 X. Li, R. Xu, X. Wei, H. Hu, S. Zhao and Y.-M. Liu, Anal. Chem., 2017, 89, 12014-12022.

108 X. Li, P. Rout, R. Xu, L. Pan, P. B. Tchounwou, Y. Ma and Y.-M. Liu, Anal. Chem., 2018, 90, 13663-13669.

109 H. Hu, S. Smith, X. Li, Z. Qian, Y. Su, M. Lin, J. Tu and Y.-M. Liu, Anal. Bioanal. Chem., 2020, 412, 1947-1954.

110 K. Song and Q. Cheng, Appl. Spectrosc. Rev., 2020, 55, 220-242.

111 N. Singhal, M. Kumar, P. K. Kanaujia and J. S. Virdi, Front. Microbiol., 2015, 6, 791.

112 F. M. Nachtigall, A. Pereira, O. S. Trofymchuk and L. S. Santos, Nat. Biotechnol., 2020, 1-6.

113 D. S. Cornett, M. L. Reyzer, P. Chaurand and R. M. Caprioli, Nat. Methods, 2007, 4, 828-833.

114 L. A. Filla, K. L. Sanders, J. B. Coulton, R. T. Filla and J. L. Edwards, Anal. Bioanal. Chem., 2019, 411, 6399-6407.

115 D. Haidas, S. Bachler, M. Köhler, L. M. Blank, R. Zenobi and P. S. Dittrich, Anal. Chem., 2019, 91, 2066-2073.

116 D. Haidas, M. Napiorkowska, S. Schmitt and P. S. Dittrich, Anal. Chem., 2020, 92, 3810-3818.

117 K. P. Law and J. R. Larkin, Anal. Bioanal. Chem., 2011, 399, 2597-2622.

118 T. R. Northen, J.-C. Lee, L. Hoang, J. Raymond, D.-R. Hwang, S. M. Yannone, C.-H. Wong and G. Siuzdak, Proc. Natl. Acad. Sci. U. S. A., 2008, 105, 3678-3683.

119 T. de Rond, J. Gao, A. Zargar, M. de Raad, J. Cunha, T. R. Northen and J. D. Keasling, Angew. Chem., 2019, 58(30), 10114-10119.

120 E. Al-Hetlani, M. O. Amin, M. Madkour and B. D'Cruz, Talanta, 2021, 221, 121556.

121 K. Deng, J. Zeng, G. Cheng, J. Gao, K. L. Sale, B. A. Simmons, A. K. Singh, P. D. Adams and T. R. Northen, Biotechnol. Biofuels, 2018, 11, 266.

122 J. Wang, M. Jie, H. Li, L. Lin, Z. He, S. Wang and J.-M. Lin, Talanta, 2017, 168, 222-229.

123 Q. Zhu, F. Teng, Z. Wang, Y. Wang and N. Lu, Anal. Bioanal. Chem., 2019, 411, 1135-1142.

124 N. Li, S. Dou, L. Feng, X. Wang and N. Lu, Talanta, 2019, 205, 120085.

125 T. R. Northen, O. Yanes, M. T. Northen, D. Marrinucci, W. Uritboonthai, J. Apon, S. L. Golledge, A. Nordström and G. Siuzdak, Nature, 2007, 449, 1033-1036.

126 J. Gao, M. de Raad, B. P. Bowen, R. N. Zuckermann and T. R. Northen, Anal. Chem., 2016, 88, 1625-1630.

127 J. A. Fincher, D. R. Jones, A. R. Korte, J. E. Dyer, P. Parlanti, A. Popratiloff, C. A. Brantner, N. J. Morris, R. K. Pirlo, V. K. Shanmugam and A. Vertes, Sci. Rep., 2019, 9, 17508.

128 A. R. Korte, N. J. Morris and A. Vertes, Anal. Chem., 2019, 91, 3951-3958.

129 A. R. Korte, S. A. Stopka, N. Morris, T. Razunguzwa and A. Vertes, Anal. Chem., 2016, 88, 8989-8996.

130 B. N. Walker, J. A. Stolee, D. L. Pickel, S. T. Retterer and A. Vertes, J. Phys. Chem. C, 2010, 114, 4835-4840.

131 H. N. Abdelhamid, Microchim. Acta, 2019, 186, 682.
132 F. Lapierre, G. Piret, H. Drobecq, O. Melnyk, Y. Coffinier, V. Thomy and R. Boukherroub, Lab Chip, 2011, 11, 1620-1628.

133 J. Heinemann, K. Deng, S. C. C. Shih, J. Gao, P. D. Adams, A. K. Singh and T. R. Northen, Lab Chip, 2017, 17, 323-331.

134 M. He, B. Chen, H. Wang and B. Hu, Appl. Spectrosc. Rev., 2019, 54, 250-263.

135 H. Wang, B. Chen, M. He and B. Hu, Anal. Chem., 2017, 89, 4931-4938.

136 H. Wang, B. Chen, M. He, X. Li, P. Chen and B. Hu, Talanta, 2019, 200, 398-407.

137 X. Ding, P. Li, S.-C. Steven Lin, Z. S. Stratton, N. Nama, F. Guo, D. Slotcavage, X. Mao, J. Shi, F. Costanzo and T. Jun Huang, Lab Chip, 2013, 13, 3626-3649.

138 T. Franke, A. R. Abate, D. A. Weitz and A. Wixforth, Lab Chip, 2009, 9, 2625-2627.

139 P. Zhang, H. Bachman, A. Ozcelik and T. J. Huang, Annu. Rev. Anal. Chem., 2020, 13, 17-43.

140 L. Song, Y. You and T. Evans-Nguyen, Anal. Chem., 2019, 91, 912-918.

141 L. Pintabona, A. Astefanei, G. L. Corthals and A. C. van Asten, J. Am. Soc. Mass Spectrom., 2019, 30, 2655-2669.

142 S. H. Yoon, Y. Huang, J. S. Edgar, Y. S. Ting, S. R. Heron, Y. Kao, Y. Li, C. D. Masselon, R. K. Ernst and D. R. Goodlett, Anal. Chem., 2012, 84, 6530-6537.

143 L. Monkkonen, J. S. Edgar, D. Winters, S. R. Heron, C. L. Mackay, C. D. Masselon, A. A. Stokes, P. R. R. Langridge-Smith and D. R. Goodlett, J. Chromatogr. A, 2016, 1439, 161-166.

144 A. Kiontke, M. Roudini, S. Billig, A. Fakhfouri, A. Winkler and C. Birkemeyer, Sci. Rep., 2021, 11, 2948.

145 D. Sun, K. F. Böhringer, M. Sorensen, E. Nilsson, J. S. Edgar and D. R. Goodlett, Lab Chip, 2020, 20, 3269-3277.

146 D. Sun, K. F. Böhringer, M. Sorensen, E. Nilsson and D. R. Goodlett, in 2019 20th International Conference on Solid-State Sensors, Actuators and Microsystems Eurosensors XXXIII (Transducers Eurosensors XXXIII), 2019, pp. 49-52.

147 Y. Zhu, P. D. Piehowski, R. Zhao, J. Chen, Y. Shen, R. J. Moore, A. K. Shukla, V. A. Petyuk, M. CampbellThompson, C. E. Mathews, R. D. Smith, W.-J. Qian and R. T. Kelly, Nat. Commun., 2018, 9, 1-10.

148 K. Rosenthal, V. Oehling, C. Dusny and A. Schmid, FEMS Microbiol. Rev., 2017, 41, 751-780.

149 Y. Li, J. D. Motschman, S. T. Kelly and B. B. Yellen, Anal. Chem., 2020, 92, 2794-2801.

150 A. R. Wheeler, W. R. Throndset, R. J. Whelan, A. M. Leach, R. N. Zare, Y. H. Liao, K. Farrell, I. D. Manger and A. Daridon, Anal. Chem., 2003, 75, 3581-3586.

151 H. Yin and D. Marshall, Curr. Opin. Biotechnol, 2012, 23, 110-119.

152 R. N. Zare and S. Kim, Annu. Rev. Biomed. Eng., 2010, 12, 187-201.

153 E. T. Jansson, T. J. Comi, S. S. Rubakhin and J. V. Sweedler, ACS Chem. Biol., 2016, 11, 2588-2595.

154 T.-H. Ong, D. J. Kissick, E. T. Jansson, T. J. Comi, E. V. Romanova, S. S. Rubakhin and J. V. Sweedler, Anal. Chem., 2015, 87, 7036-7042. 
155 T. J. Comi, M. A. Makurath, M. C. Philip, S. S. Rubakhin and J. V. Sweedler, Anal. Chem., 2017, 89, 7765-7772.

156 S. K. Küster, M. Pabst, R. Zenobi and P. S. Dittrich, Angew. Chem., Int. Ed., 2015, 54, 1671-1675.

157 S. K. Küster, S. R. Fagerer, P. E. Verboket, K. Eyer, K. Jefimovs, R. Zenobi and P. S. Dittrich, Anal. Chem., 2013, 85, 1285-1289.

158 A. J. Ibáñez, S. R. Fagerer, A. M. Schmidt, P. L. Urban, K. Jefimovs, P. Geiger, R. Dechant, M. Heinemann and R. Zenobi, Proc. Natl. Acad. Sci. U. S. A., 2013, 110, 8790-8794.

159 O. Guillaume-Gentil, T. Rey, P. Kiefer, A. J. Ibáñez, R. Steinhoff, R. Brönnimann, L. Dorwling-Carter, T. Zambelli, R. Zenobi and J. A. Vorholt, Anal. Chem., 2017, 89, 5017-5023.

160 Q. Huang, S. Mao, M. Khan, W. Li, Q. Zhang and J.-M. Lin, Chem. Sci., 2019, 11, 253-256.

161 F. H. Arnold, Acc. Chem. Res., 1998, 31, 125-131.

162 R. Baran, W. Reindl and T. R. Northen, Curr. Opin. Microbiol., 2009, 12, 547-552.

163 X. W. Diefenbach, I. Farasat, E. D. Guetschow, C. J. Welch, R. T. Kennedy, S. Sun and J. C. Moore, ACS Omega, 2018, 3, 1498-1508.

164 S. Carillo, C. Jakes and J. Bones, J. Pharm. Biomed. Anal., 2020, 185, 113218.

165 S. J. B. Dunham, J. F. Ellis, B. Li and J. V. Sweedler, Acc. Chem. Res., 2017, 50, 96-104.

166 R. D. Holland, J. G. Wilkes, F. Rafii, J. B. Sutherland, C. C. Persons, K. J. Voorhees and J. O. Lay, Rapid Commun. Mass Spectrom., 1996, 10, 1227-1232.

167 J. O. Lay and R. D. Holland, in Mass Spectrometry of Proteins and Peptides: Mass Spectrometry of Proteins and Peptides, ed. J. R. Chapman, Humana Press, Totowa, NJ, 2000, pp. 461-487.

168 T. L. Simmons, R. C. Coates, B. R. Clark, N. Engene, D. Gonzalez, E. Esquenazi, P. C. Dorrestein and W. H. Gerwick, Proc. Natl. Acad. Sci. U. S. A., 2008, 105, 4587-4594.

169 T. Si, B. Li, T. J. Comi, Y. Wu, P. Hu, Y. Wu, Y. Min, D. A. Mitchell, H. Zhao and J. V. Sweedler, J. Am. Chem. Soc., 2017, 139, 12466-12473.

170 C. Dusny, M. Lohse, T. Reemtsma, A. Schmid and O. J. Lechtenfeld, Anal. Chem., 2019, 91, 7012-7018.

171 X. Feng, B.-F. Liu, J. Li and X. Liu, Mass Spectrom. Rev., 2015, 34, 535-557.

172 W. Wu, D. Zhang, K. Chen, P. Zhou, M. Zhao, L. Qiao and B. Su, Anal. Chem., 2018, 90, 14395-14401.

173 J. Wang, P. H. Chao and R. Michael van Dam, Proceedings of the 23rd International Conference on Miniaturized Systems for Chemistry and Life Sciences (MicroTAS), Basel, Switzerland, 2019, pp. 546-547.

174 J. Kehe, A. Kulesa, A. Ortiz, C. M. Ackerman, S. G. Thakku, D. Sellers, S. Kuehn, J. Gore, J. Friedman and P. C. Blainey, Proc. Natl. Acad. Sci. U. S. A., 2019, 201900102.
175 G. Sathyanarayanan, M. Haapala and T. Sikanen, Micromachines, 2018, 9, 649.

176 I. Sinclair, M. Bachman, D. Addison, M. Rohman, D. C. Murray, G. Davies, E. Mouchet, M. E. Tonge, R. G. Stearns, L. Ghislain, S. S. Datwani, L. Majlof, E. Hall, G. R. Jones, E. Hoyes, J. Olechno, R. N. Ellson, P. E. Barran, S. D. Pringle, M. R. Morris and J. Wingfield, Anal. Chem., 2019, 91, 3790-3794.

177 SCIEX Ltd, Echo MS | SCIEX, https:/sciex.com/products/ integrated-solutions/echo-ms?utm_source=adwords+search\& utm_medium $=$ cpc\&utm_campaign $=2020+$ product + launches\& utm_term=g-b-h-a-echo+ms+search+ads-may-20\&utm_content= echo+ms\&gclid=EAIaIQobChMI8NCQ0vm56gIVmx-tBh0wTAqw EAAYASAAEgKZtvD_BwE, accessed 15 October 2020.

178 J. Rhys, E. Hoyes, E. Sprake, P. DeLand, L. Ghislain, E. Hall, S. Datwani, I. Sinclair, M. Bachmann and J. Wingfield, Waters corporation, https://www.labcyte.com/media/pdf/ POS-Waters-AZ-Labcyte-Acoustic-Mist-Ionisation.pdf.

179 W. Xu, N. E. Manicke, G. R. Cooks and Z. Ouyang, JALA, 2010, 15, 433-439.

180 D. T. Snyder, C. J. Pulliam, Z. Ouyang and R. G. Cooks, Anal. Chem., 2016, 88, 2-29.

181 L. Li, T.-C. Chen, Y. Ren, P. I. Hendricks, R. G. Cooks and Z. Ouyang, Anal. Chem., 2014, 86, 2909-2916.

182 Z. Ouyang and R. G. Cooks, Annu. Rev. Anal. Chem., 2009, 2, 187-214.

183 P. I. Hendricks, J. K. Dalgleish, J. T. Shelley, M. A. Kirleis, M. T. McNicholas, L. Li, T.-C. Chen, C.-H. Chen, J. S. Duncan, F. Boudreau, R. J. Noll, J. P. Denton, T. A. Roach, Z. Ouyang and R. G. Cooks, Anal. Chem., 2014, 86, 2900-2908.

184 G. A. Eiceman, TrAC, Trends Anal. Chem., 2002, 21, 259-275.

185 E. J. Davis, P. Dwivedi, M. Tam, W. F. Siems and H. H. Hill, Anal. Chem., 2009, 81, 3270-3275.

186 E. C. A. Stigter, G. J. de Jong and W. P. van Bennekom, TrAC, Trends Anal. Chem., 2013, 45, 107-120.

187 S. Joshi, H. Zuilhof, T. A. van Beek and M. W. F. Nielen, Anal. Chem., 2017, 89, 1427-1432.

188 P. W. Fedick, F. Pu, N. M. Morato and R. G. Cooks, J. Am. Soc. Mass Spectrom., 2020, 31, 735-741.

189 M. Hoffmann, H. Kleine-Weber, S. Schroeder, N. Krüger, T. Herrler, S. Erichsen, T. S. Schiergens, G. Herrler, N.-H. Wu, A. Nitsche, M. A. Müller, C. Drosten and S. Pöhlmann, Cell, 2020, 181, 271-280.e8.

190 A. M. Klein, L. Mazutis, I. Akartuna, N. Tallapragada, A. Veres, V. Li, L. Peshkin, D. A. Weitz and M. W. Kirschner, Cell, 2015, 161, 1187-1201.

191 J. Grant, S. H. Goudarzi and M. Mrksich, Anal. Chem., 2018, 90, 13096-13103.

192 Y. Zhao, M. Tang, F. Liu, H. Li, H. Wang and D. Xu, Anal. Chem., 2019, 91, 13418-13426. 